\title{
Spinodal decomposition in a mean-field model of the cortex: Emergence of hexagonally symmetric activation patterns
}

\author{
Moira L. Steyn-Ross ${ }^{*}$ and D. A. Steyn-Ross \\ School of Engineering, University of Waikato, Hamilton, New Zealand \\ L. J. Voss \\ Waikato Hospital, Hamilton, New Zealand \\ J. W. Sleigh \\ Waikato Clinical School, University of Auckland, Waikato Hospital, Hamilton, New Zealand
}

(Received 27 August 2017; revised manuscript received 20 October 2018; published 17 January 2019)

\begin{abstract}
Spinodal decomposition is a well-known pattern-forming mechanism in metallurgic alloys, semiconductor crystals, and colloidal gels. In metallurgy, if a heated sample of a homogeneous $\mathrm{Zn}$-Al alloy is suddenly quenched below a critical temperature, then the sample can spontaneously precipitate into inhomogenous textures of $\mathrm{Zn}$ - and Al-rich regions with significantly altered material properties such as ductility and hardness. Here we report on our recent discovery that a two-dimensional model of the human cortex with inhibitory diffusion can, under particular homogeneous initial conditions, exhibit a form of nonconserved spinodal decomposition in which regions of the cortex self-organize into hexagonally distributed binary patches of activity and inactivity. Fine-scale patterns precipitate rapidly, and then the dynamics slows to render coarser-scale shapes which can ripen into a range of slowly evolving patterns including mazelike labyrinths, hexagonal islands and continents, nucleating "mitotic cells" which grow to a critical size then subdivide, and inverse nucleations in which quiescent islands are surrounded by a sea of activity. One interesting class of activity coalesces into a soliton-like narrow ribbon of depolarization that traverses the cortex at $\sim 4 \mathrm{~cm} / \mathrm{s}$. We speculate that this may correspond to the thus far unexplained interictal waves of cortical activation that precede grand-mal seizure in an epileptic event. We note that spinodal decomposition is quite distinct from the Turing mechanism for symmetry breaking in cortex investigated in earlier work by the authors [Steyn-Ross et al., Phys. Rev. E 76, 011916 (2007)].
\end{abstract}

DOI: 10.1103/PhysRevE.99.012318

\section{INTRODUCTION}

In his ground-breaking morphogenesis paper, Turing showed that a chemical system comprised of a homogenous mixture of interacting "activator" and "inhibitor" species could spontaneously organize into heterogenous spatial patterns [1]. Now referred to as the Turing instability, this mechanism for pattern formation has been studied in many chemical reaction-diffusion systems (e.g., Refs. [2-4]), and it is now well established that these spatial structures can only emerge when the level of inhibitory diffusion is sufficiently strong.

Competitive diffusions have been proposed as a mechanism for pattern formation in the brain. Wilson and Cowan's 1973 paper [5] showed that stationary neural activity patterns can form in a cortical network of excitatory and inhibitory neurons provided that the inhibitory axons have a longer

\footnotetext{
*msr@waikato.ac.nz

Published by the American Physical Society under the terms of the Creative Commons Attribution 4.0 International license. Further distribution of this work must maintain attribution to the author(s) and the published article's title, journal citation, and DOI.
}

spatial range than the excitatory axons: Short-range recurrent excitation stabilizes local neural activity while longer range inhibition prevents local activity from spreading. This dominance of distal inhibition is referred to as lateral inhibition and is frequently implemented as a "Mexican hat" connectivity kernel featuring long-range inhibitory and short-range excitatory axonal connections. There is an extensive and very active literature exploring spatial bifurcations in Wilson-Cowan-like mean-field neural models [6-12].

An alternative mechanism for Turing instability in the cortex was proposed by Steyn-Ross et al. [13,14]. Rather than imposing lateral inhibition via a shaped connectivity kernel for chemical synapses, the authors suggested that long-range inhibition arises naturally from the relative distribution of $E-E$ and $I-I$ gap-junction synapses that form direct ohmic connections between adjoining pairs of excitatory $(E)$ or inhibitory $(I)$ neurons. In contrast to the very sparse $E-E$ gap junctions, $I-I$ junctions between neighboring interneurons are ubiquitous throughout the cortex and are supposed to form an inhibitory diffusive syncytium that scaffolds the brain $[15,16]$. Using a noise-driven two-dimensional (2D) continuum model of the cortex, the authors showed how gap-junction connectivities could be mapped to excitatory and inhibitory diffusion strengths $D_{1}$ and $D_{2}$, respectively, and found that, provided 
$D_{2} \gg D_{1}$ with $D_{2}$ sufficiently strong, a previously stable equilibrium state could become unstable, leading to the emergence of nonequilibrium Turing-structured states [13].

In this paper we propose a new mechanism for pattern formation in the cortex: spinodal decomposition. This is a spontaneous phase separation process that is well known in the metallurgy and colloidal literature. In this diffusion-driven process, a stable homogeneous mixture of species $A$ and $B$ is rendered thermodynamically unstable by suddenly cooling the mixture below its critical temperature. This abrupt temperature reduction, known as a quench, causes the originally wellmixed state to spontaneously unmix into separated regions of A-rich and B-rich phases. The initial decomposition proceeds exponentially fast and then slows as the patterns mature. The first theoretical explanation for temperature-induced phase separation in alloys was given by Cahn $[17,18]$, who coined the phrase "spinodal decomposition." Since its discovery in metallurgy, spinodal decomposition has since been implicated as a mechanism for spatial self-organization in polymers [19,20], surface waves [21], Abrikosov vortex lattices in superconductors [22], dendrite growth [23], quantum dots [24], and colloidal crystals [25].

In the cortex, assemblies of neurons can be either actively firing or quiescent, so we interpret these two distinct states as representing the two alternate phases of cortical activity. For our model, we find that the level of cortical responsiveness is determined by a delicate dynamic balance between external excitatory and inhibitory influences: Too little excitation (or too strong an inhibition) leads to coma, while unconstrained excitation leads to seizure. We take the net excitatory-inhibitory $(E, I)$ balance in the cortex to be analogous to the physical temperature of, say, a metallic alloy or an oil-water mixture.
From previous work, we have found that the model cortex is most responsive to input stimulus when it is positioned close to or within the region of state space in which the cortex has access to multiple steady states as indicated in Fig. 1 (left panel). The three-state region is shown as a narrowing coppercolored wedge running northwest to southeast, terminating at the critical point (CP: red circle) marking the codimension-2 cusp at which the three roots coalesce to a single solution. The $(E, I)$ balance is determined by the selected $\left(\Delta V_{e}^{\text {rest }}, \lambda_{i}\right)$ coordinate: $\Delta V_{e}^{\text {rest }}$ is the perturbation to the resting voltage of the excitatory neural population, and $\lambda_{i}$ is the synaptic gain of the inhibitory population (see Sec. II A for model details).

To organize a "thermodynamic quench," we might select a one-root coordinate lying just beyond the critical point to represent the homogeneous ("well-mixed") initial state, then quench by abruptly translating to a coordinate inside the multiroot region. Provided that the quench terminates at the unstable midbranch solution, and that the upper and lower branches are stable, then spontaneous decomposition into high- and low-firing patches of cortex can occur. We note that both Turing and spinodal mechanisms require that the cortex be located within the multiple-root coexistence region that supports high-, mid-, and low-firing equilibrium states. But unlike the Turing mechanism which evolves from a spatially destabilized upper or lower branch, spinodal decomposition proceeds from the always unstable midbranch. The homogeneous phase promptly destabilizes, precipitating simultaneously onto the upper (high-firing, active) and lower (low-firing, quiescent) equilibrium branches, forming patterns that develop rapidly and then more slowly as the patterns mature.

The paper is structured as follows. In Sec. II, we define the cortical model, locate its equilibrium states, and plot
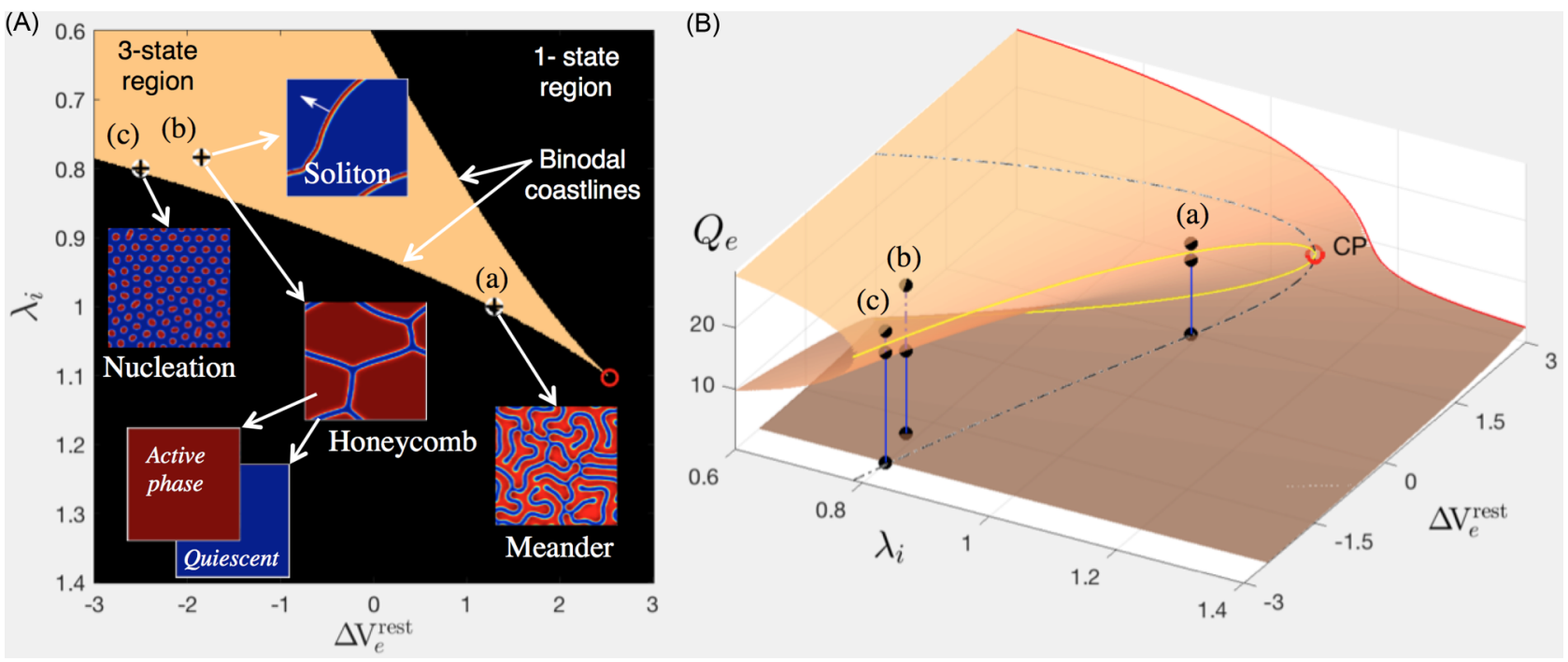

FIG. 1. Manifold of cortical equilibrium states. (A) Bird's-eye view showing the three-state regions in copper (light gray) and single-state surroundings (black) as a function of inhibitory gain $\lambda_{i}$ and excitatory offset $\Delta V_{e}^{\text {rest }}$. The coexistence region is bounded by the "binodal coastlines" in (A) and by the solid-yellow (light gray) and dot-dashed black curves in (B). The red (medium-gray) circle marks critical point CP. The three $(\oplus)$ markers labeled (a), (b), and (c) locate the $\left(\Delta V_{e}^{\text {rest }}, \lambda_{i}\right)$ coordinate resulting in the spinodal decompositions illustrated in the meander, honeycomb, soliton, and nucleation panels. (B) Three-dimensional view of distribution of excitatory firing rates $Q_{e}$ across the $\lambda_{i}-\Delta V_{e}^{\text {rest }}$ domain. The (a), (b), and (c) simulations all lie within the binodal multiroot region characterized by a stable dark-red (midgray) upper "active phase" and deep-blue (dark-gray) lower "quiescent" branch, separated by an unstable midbranch. All simulations are initialized to commence at the unstable midbranch equilibrium point. 
the wave-number-dependent linear stability predictions for four representative spinodal decomposition simulations. In Sec. III, we report the outcomes of a range of stochastic numerical simulations on a $240 \times 240$ cortical grid. We demonstrate that the morphology of the emerging spinodal patterns is sensitive to the choice of $\left(\Delta V_{e}^{\text {rest }}, \lambda_{i}\right)$ coordinate by showing formation of hexagonal, labyrinthine, and nucleating cellular arrays of cortical activity. Despite their disparate shapes, we find that the 2D spatial spectra for the matured stationary patterns reveal a common hexagonal basis. Analysis of the time-dependent decomposition allows us to distinguish three stages of pattern formation: rapid precipitation and differentiation within $\sim 1 \mathrm{~s}$ and then slow maturation over tens of seconds. By reducing the inhibitory rate constant, we show that the stationary hexagonal pattern can be destabilized by the presence of a marginally damped Hopf bifurcation, leading to the unexpected formation of a slow-moving soliton shockfront. We argue that this might correspond to the interictal waves of excitation commonly seen prior to the onset of seizure activity in cortex. In Sec. IV, we offer provide a commentary about how spinodal decomposition might relate to hexagonally organized activity patterns that have been observed or postulated in the brain.

\section{METHODS}

\section{A. Model equations}

We model the cortex as a 2D mean-field continuum of excitatory and inhibitory neurons that are interconnected locally via resistive gap junctions and neurotransmitter-mediated chemical synapses and over distance via long-range myelinated axons. Since scalp EEG (electroencephalogram) electrodes can only sense population-average voltages, cortical parameters have been coarse grained over a spatial extent of order $1 \mathrm{~mm}^{2}$, corresponding to the area of a cortical macrocolumn [26].

The spatially averaged excitatory and inhibitory soma potentials $V_{e}$ and $V_{i}$ at grid location $\vec{r}=(x, y)$ obey the following equations of motion:

$$
\begin{aligned}
\tau_{b} \frac{\partial V_{b}(\vec{r}, t)}{\partial t}= & V_{b}^{\text {rest }}+\Delta V_{b}^{\text {rest }}-V_{b}(\vec{r}, t)+\left[\rho_{e} \psi_{e b}(\vec{r}, t) \Phi_{e b}(\vec{r}, t)\right. \\
& \left.+\rho_{i} \psi_{i b}(\vec{r}, t) \Phi_{i b}(\vec{r}, t)\right]+D_{b b} \nabla^{2} V_{b}(\vec{r}, t) \\
& \text { with } \quad b=e, i
\end{aligned}
$$

where we follow a left-to-right convention for labeling pre-to-postsynaptic connectivity. The terms [...] in square brackets are chemical-synaptic voltage inputs. The $\nabla^{2}$ symbol denotes the 2D Laplacian operator $\nabla^{2} \equiv\left(\partial^{2} / \partial x^{2}+\partial^{2} / \partial y^{2}\right)$ for the resistive gap-junction (GJ) diffusion inputs with excitatory strength $D_{e e} \equiv D_{1}$ for $E-E$ connections and inhibitory strength $D_{i i} \equiv D_{2}$ for $I-I$. Because inhibitory GJs are substantially more abundant than excitatory GJs in cortical tissue [13,27], we set $D_{1}$ to be a small fraction of $D_{2}\left(D_{1}=D_{2} / 100\right)$.

The $\tau_{b}$ in Eq. (1) are soma time constants for the neuron populations and $V_{b}^{\text {rest }}$ their respective resting voltages; see Table I for values. The $\rho_{b}$ are the chemical synaptic gains given by the area under the unitary postsynaptic potential,

\begin{tabular}{|c|c|c|c|}
\hline Symbol & Description & Value & Unit \\
\hline$\Delta V_{e}^{\text {rest }}$ & Offset to excitatory resting potential & -2.5 to +2.5 & $\mathrm{mV}$ \\
\hline$\lambda_{i}$ & Inhibitory scale factor & $0.78-1.10$ & - \\
\hline$D_{2}$ & $I \leftrightarrow I$ gap-junction diffusive coupling & $0.30-0.45$ & $\mathrm{~cm}^{2}$ \\
\hline$\gamma_{i}$ & Inhibitory rate constant, $\gamma_{i}=\gamma_{i}^{0} / \lambda_{i}$ & $\gamma_{i}^{0}=22-80$ & $\mathrm{~s}^{-1}$ \\
\hline$\Lambda_{e b}$ & Inverse length scale for $E \rightarrow b$ axonal connections & $4-28$ & $\mathrm{~cm}^{-1}$ \\
\hline$V_{e, i}^{\text {rest }}$ & Neuron resting potential & $-64,-64$ & $\mathrm{mV}$ \\
\hline$D_{1}$ & $E \leftrightarrow E$ gap-junction diffusive coupling & $D_{2} / 100$ & $\mathrm{~cm}^{2}$ \\
\hline$\gamma_{e}$ & Excitatory rate constant & 170 & $\mathrm{~s}^{-1}$ \\
\hline$\tau_{e, i}$ & Neuron time constant & $0.040,0.040$ & $\mathrm{~s}$ \\
\hline$V_{e, i}^{\text {rev }}$ & Reversal potential for (AMPA, GABA) receptors & $0,-70$ & $\mathrm{mV}$ \\
\hline$\rho_{e}$ & Excitatory synaptic gain & $1.00 \times 10^{-3}$ & $\mathrm{mV} \mathrm{s}$ \\
\hline$\rho_{i}$ & Inhibitory synaptic gain, $\rho_{i}=\lambda_{i} \rho_{i}^{0}$ & $\rho_{i}^{0}=-1.05 \times 10^{-3}$ & $\mathrm{mV} \mathrm{s}$ \\
\hline$N_{e b}^{\alpha}$ & Long-range $E \rightarrow b$ axonal connectivity & 2000 & - \\
\hline$N_{e b, i b}^{\beta}$ & Local $E \rightarrow b, I \rightarrow b$ axonal connectivity & 800,600 & - \\
\hline$\left\langle\phi_{e b}^{\mathrm{sc}}\right\rangle$ & $E \rightarrow b$ tonic flux entering from subcortex & 300 & $\mathrm{~s}^{-1}$ \\
\hline$s_{0}$ & Subcortical noise scale-factor (default value) & 0.125 & - \\
\hline$v$ & Axonal conduction speed & 140 & $\mathrm{~cm} \mathrm{~s}^{-1}$ \\
\hline$Q_{e, i}^{\max }$ & Maximum firing rate & 30,60 & $\mathrm{~s}^{-1}$ \\
\hline$\theta_{e, i}$ & Sigmoid threshold voltage & $-58.5,-58.5$ & $\mathrm{mV}$ \\
\hline$\sigma_{e, i}$ & Standard deviation for threshold & 3,5 & $\mathrm{mV}$ \\
\hline
\end{tabular}
with $\rho_{e}>0$ and $\rho_{i}<0$. These synaptic gains are scaled by

TABLE I. Symbol definitions and standard values for cortical model.

Note. Our spinodal simulations apply changes to the five parameters listed at the top of the table: Static excitatory-inhibitory balance is set by the $\left(\Delta V_{e}^{\text {rest }}, \lambda_{i}\right)$ coordinate; dynamical properties are by controlled by $D_{2}$ and $\Lambda_{e b}$ (spatial stability) and by $\gamma_{i}$ (temporal stability). Subscript label $b$ indicates that destination can be either of type $E$ (excitatory) or $I$ (inhibitory). Values modified from Table 15.1 in Steyn-Ross et al. [28]. 
dimensionless reversal-potential functions $\psi_{a b}$,

$$
\psi_{a b}(\vec{r}, t)=\frac{V_{a}^{\mathrm{rev}}-V_{b}(\vec{r}, t)}{V_{a}^{\mathrm{rev}}-V_{b}^{\mathrm{rest}}},
$$

that are normalized to unity when the neuron is at resting voltage and are zero when the membrane voltage reaches the relevant reversal potential, taken to be $V_{e}^{\text {rev }}=0 \mathrm{mV}$ for excitatory (AMPA) receptors and $V_{i}^{\text {rev }}=-70 \mathrm{mV}$ for inhibitory (GABA) receptors.

The four $\Phi_{a b}$ functions in Eq. (1) are postsynaptic fluxes governed by second-order differential equations,

$$
\begin{aligned}
& \left(\frac{\partial}{\partial t}+\gamma_{e}\right)^{2} \Phi_{e b}(\vec{r}, t) \\
& \quad=\gamma_{e}^{2}\left[N_{e b}^{\alpha} \phi_{e b}^{\alpha}(\vec{r}, t)+N_{e b}^{\beta} Q_{e}(\vec{r}, t)+\phi_{e b}^{\mathrm{sc}}(\vec{r}, t)\right], \\
& \left(\frac{\partial}{\partial t}+\gamma_{i}\right)^{2} \Phi_{i b}(\vec{r}, t)=\gamma_{i}^{2} N_{i b}^{\beta} Q_{i}(\vec{r}, t),
\end{aligned}
$$

whose impulse responses are alpha functions of the form $\gamma^{2} t \exp (-\gamma t) ; \gamma$ is the rate constant and $1 / \gamma$ the time-topeak. Connectivities $N_{e b}^{\alpha}$ and $N_{e b}^{\beta}$ are the number of longand short-range input connections via chemical synapses, with $\phi_{e b}^{\alpha}, Q_{e, i}$ being the corresponding long-range and local spike-rate fluxes. The long-range incoming flux of spike-rate activity $\phi_{e b}^{\alpha}$ obeys a 2D damped wave equation [29],

$$
\left[\left(\frac{\partial}{\partial t}+v \Lambda_{e b}\right)^{2}-v^{2} \nabla^{2}\right] \phi_{e b}^{\alpha}(\vec{r}, t)=\left(v \Lambda_{e b}\right)^{2} Q_{e}(\vec{r}, t),
$$

where $\Lambda_{e b}$ is the inverse length scale for $E \rightarrow b$ axonal connections and $v$ is the axonal conduction speed. $Q(\vec{r}, t)$ is the source of spike-rate flux, assumed to be a sigmoidal function of membrane voltage,

$$
Q_{a}(\vec{r}, t)=\frac{Q_{a}^{\max }}{1+\exp \left[-C\left(V_{a}(\vec{r}, t)-\theta_{a}\right) / \sigma_{a}\right]},
$$

with subscript $a$ standing for $e$ [Eq. (2a)] or $i$ [Eq. (2b)] and $C=\pi / \sqrt{3}$. Here $\theta_{a}$ is the population-average threshold for firing, $\sigma_{a}$ is its standard deviation, and $Q_{a}^{\max }$ is the maximum firing rate.

The final source term on the right of Eq. (2a) represents unstructured stimulatory tone arriving from subcortical sources. This stimulus is modeled as a low-intensity spatiotemporal white-noise variation $\xi_{e b}$ about a constant tonic background $\left\langle\phi_{e b}^{\mathrm{sc}}\right\rangle$,

$$
\left.\phi_{e b}^{\mathrm{sc}}(\vec{r}, t)=\left\langle\phi_{e b}^{\mathrm{sc}}\right\rangle+s \sqrt{\left\langle\phi_{e b}^{\mathrm{sc}}\right.}\right\rangle \xi_{e b}(\vec{r}, t),
$$

with $s$ being a dimensionless amplitude scale factor. The $\xi_{e b}(\vec{r}, t)$ is Gaussian-distributed delta-correlated noise with statistics $\langle\xi(\vec{r}, t)\rangle=0$ and $\left\langle\xi_{m}\left(t_{1}\right) \xi_{n}\left(t_{2}\right)\right\rangle=\delta_{m, n} \delta\left(t_{1}-t_{2}\right)$. In numerical simulation on a $2 \mathrm{D}$ rectangular sheet with grid resolution $(\Delta x, \Delta y)$ and fixed time step $\Delta t$, we approximate white noise $\xi(\vec{r}, t)$ at grid position $\vec{r}=(x, y)=(i \Delta x, j \Delta y)$ and time $t=k \Delta t$ with discrete noise samples $\left\{\xi_{i j}^{k}\right\}$ constructed using the MATLAB randn random number generator: $\xi_{i j}^{k}=\operatorname{randn} /(\Delta x \Delta y \Delta t)^{1 / 2}$. This construction ensures that fluctuation statistics are largely independent of our choices for spatial and temporal resolutions-provided, of course, that the standard Courant-Friedrichs-Lewy stability criterion is satisfied - with the noise approximation converging to exact spatiotemporal white noise in the limit $(\Delta x, \Delta y, \Delta t) \rightarrow 0$.

\section{B. Equilibrium states of the cortex}

We assume that the homogeneous equilibrium states of the model cortex provide a reference substrate that constrains spatiotemporal cortical dynamics; of particular interest are regions of state space that support multiple steady states, since transitions between states become possible.

We locate the homogenous equilibrium states by zeroing all space- and time-derivatives in Eqs. (1)-(3) (i.e., $\nabla^{2}=0$; $\partial / \partial t=\partial^{2} / \partial t^{2}=0$ ), and zeroing the noise amplitude terms, and then solving numerically for the steady-state firing rates $\left(Q_{e}, Q_{i}\right)$ of the excitatory and inhibitory neural populations. This process is repeated across a finely spaced grid of $\left(\Delta V_{e}^{\text {rest }}, \lambda_{i}\right)$ values representing a $2 \mathrm{D}$ variation in excitatoryinhibitory $(E-I)$ cortical balance. As visualized in Fig. 1, we identify a wedge-shaped subset of the $E-I$ domain in which every $\left(\Delta V_{e}^{\text {rest }}, \lambda_{i}\right)$ coordinate can be mapped to three steady states: a stable high-firing upper-branch node, a stable low-firing quiescent lower-branch node, and an intermediatebranch separatrix that is universally unstable (as illustrated in the dispersion curves of Fig. 2).

Figure 1 highlights the three selected coordinates used in the four computer simulations (meander, honeycomb, soliton, and nucleation) that will be reported in Sec. III. It is significant to note that all four simulations are located within the threestate region bounded by the "binodal coastlines" shown in Fig. 1 (left panel), with each simulation being launched from the unstable separatrix.

\section{Linear stability predictions for the model cortex}

Noting the parameter symmetries evident in Table I $\left(N_{e e}^{\alpha}=N_{e i}^{\alpha} ; N_{e e}^{\beta}=N_{e i}^{\beta} ; N_{i e}^{\beta}=N_{i i}^{\beta}\right)$, cortical equations (1)-(3) are equivalent to eight first-order differential equations. We define the eight-variable state vector $\vec{X}=\left[V_{e}, V_{i}, \Phi_{e b}, \dot{\Phi}_{e b}\right.$, $\left.\Phi_{i b}, \dot{\Phi}_{i b}, \phi_{e b}, \dot{\phi}_{e b}\right]^{T}$ and express the cortical state as its equilibrium value $\vec{X}^{(0)}$ plus a small plane-wave perturbation $\delta \vec{X}$,

$$
\delta \vec{X}(t, \vec{r})=\delta \vec{X}(t) e^{i \vec{q} \cdot \vec{r}}=\delta \vec{X}(0) e^{\Lambda t} e^{i \vec{q} \cdot \vec{r}}
$$

with wave vector $\vec{q}$ and wave number $|\vec{q}|=q ; \Lambda$ is an eigenvalue whose real part gives the growth rate of the $\delta \vec{X}(0)$ initial perturbation: If $\operatorname{Re}(\Lambda)>0$, then an instability is predicted. Substituting $\vec{X}=\vec{X}^{(0)}+\delta \vec{X}$ into Eqs (1)-(3) and retaining only linear terms gives the matrix equation,

$$
\frac{d}{d t} \delta \vec{X}=\mathbf{J}(q) \delta \vec{X},
$$

where $\mathbf{J}$ is an $8 \times 8$ Jacobian matrix in which the $\nabla^{2}$ Laplacians for diffusion [Eq. (1)] and wave propagation [Eq. (3)] appear as $-q^{2}$ terms. For each wave number $q$, we extract and plot the dominant eigenvalue-i.e., that eigenvalue whose real part is most positive (or least negative) - since this describes the most strongly growing (or most long-lived) mode at a given spatial frequency.

Figure 2 plots the homogeneous linear stability predictions for the four simulation experiments indicated in Fig. 1 and 
(a) Meander

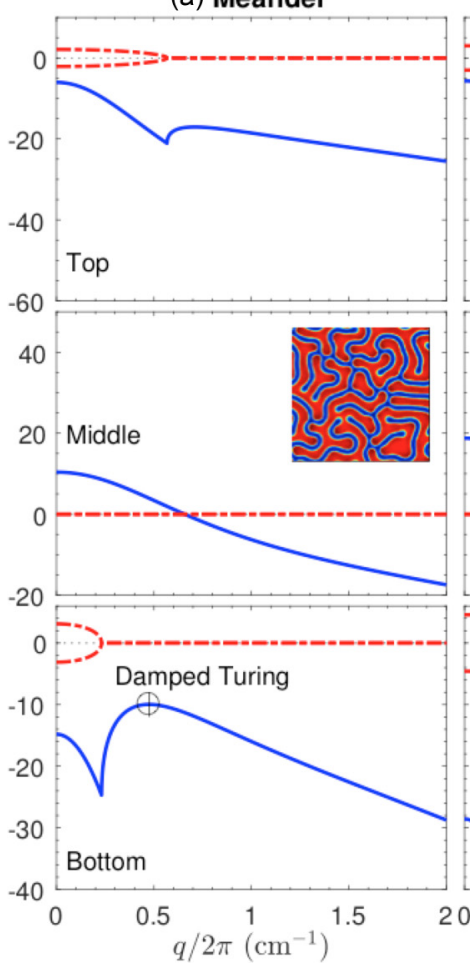

(b) Nucleation

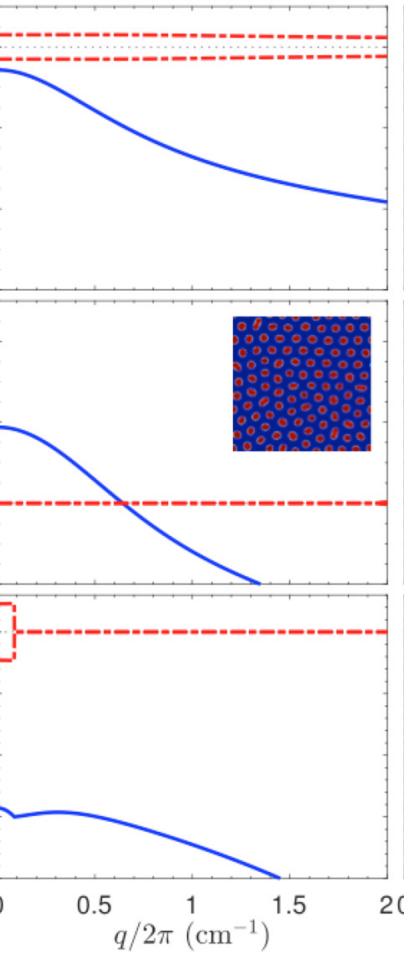

(c) Honeycomb

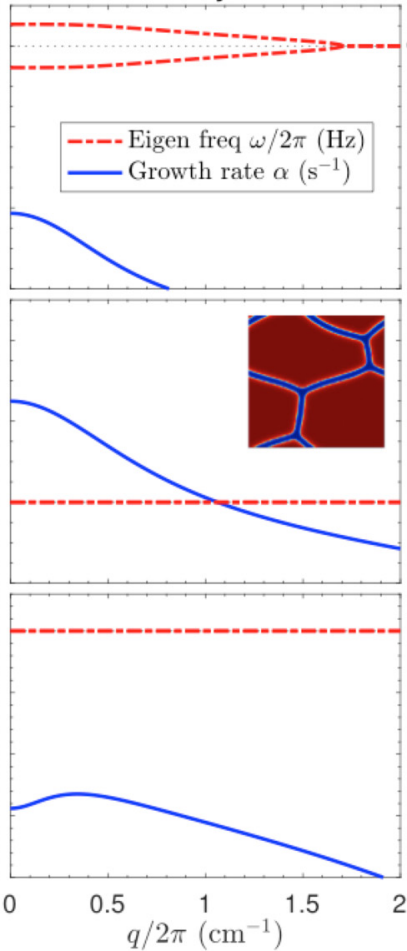

(d) Soliton

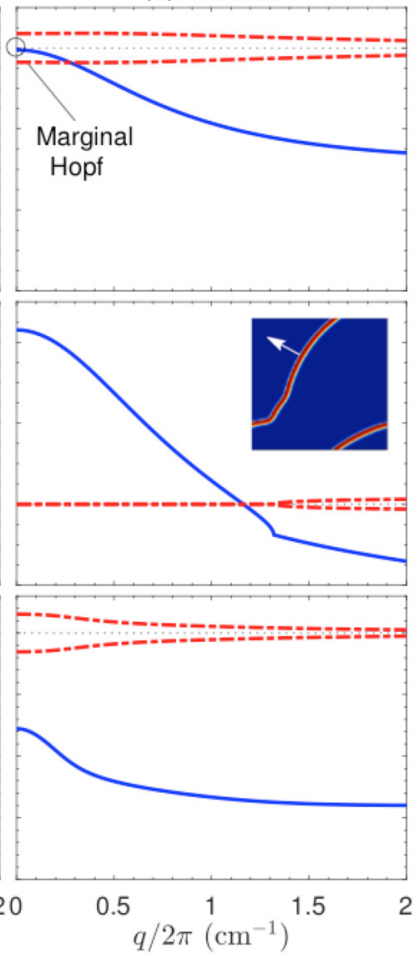

FIG. 2. Linear stability predictions as a function of wave number for the four decomposition simulations indicated in Fig. 1 and captured in the (a) meander (Fig. 5), (b) nucleation (Fig. 6), (c) honeycomb (Fig. 4), and (d) soliton (Fig. 10) snap-shot galleries. From top to bottom, panel rows display, respectively, the dispersion trends for the top, middle, and bottom homogeneous equilibrium states. Dispersion graphs have been ordered from left to right to show progressive increases in the degree of instability of the middle branch. Solid-blue (black) curves show growth rate $\alpha$ (in $\mathrm{s}^{-1}$ ) predicted from linear eigenvalue analysis, with $\alpha(q)>0$ indicating instability at wave number $q$; dashed-red (dashed-gray) curves show corresponding $\omega(q) / 2 \pi$ (Hz) eigenfrequency trends. In all cases, top- and bottom-branch equilibria are stable across all wave numbers, although the top branch for (d) soliton is very close to a 3.5-Hz Hopf bifurcation $\left(\alpha=-0.001 \mathrm{~s}^{-1}\right.$ at zero wave number). The emergent Turing-like labyrinth structure in (a) seems to be guided by the presence of a damped Turing bifurcation $(\oplus$ ) on the bottom branch near $q / 2 \pi \approx 0.5 \mathrm{~cm}^{-1}$.

later explored in Sec. III. In all cases the top and bottom branches are stable (i.e., growth rate $\alpha=\operatorname{Re}(\Lambda)<0$ for all wave numbers $q$ ) -although this is only marginally true for the upper-branch soliton setting (top-right panel). Negative growth rates mean that small perturbations away from steady state (on either the top or bottom branch) will decay back to the homogeneous equilibrium substrate, and thus no emergent Turing or wave patterns are expected for simulations launched from either stable node.

In contrast, the midbranch separatrix is strongly unstable in all cases, with maximum growth predicted at $q=0$, i.e., the homogeneous state is maximimally unstable. Therefore a simulation launched from the homogeneous separatrix will rapidly evolve into inhomogeneous patches of higher and lower activity, and these will be attracted toward the high- and low-firing stable nodes. This is the fundamental mechanism for spontaneous phase separation-and subsequent cortical pattern formation-via spinodal decomposition.

\section{Linking cortical pattern formation to thermodynamic spinodal decomposition}

In the standard Ginzburg-Landau [30] analysis of the spinodal decomposition of a metallurgic binary alloy, the free energy $g(c)$ of the binary mixture is expressed as a function of order parameter $c$, the fractional concentration of one of the two constituent phases. For the cartoon shown in Fig. 3(a), we have hypothesized that it is possible to map the binary metallurgic order parameter $(c)$ to a neuronal firing rate $(Q)$ in the cortical system, with high or low concentrations corresponding to high or low firing rates and that there exists a corresponding free energy function $g(Q)$ whose shape depends on a cortical "temperature" $T$. If the temperature exceeds a critical value (upper curve: $T_{2}>T_{c}$ ), then the free energy has a single minimum corresponding to the homogeneous well-mixed state $Q=Q_{\text {mid }}$. But if the temperature is lowered below critical (lower curve: $T_{1}<T_{c}$ ), the energy graph deforms into a double-well potential allowing separation into distinct phases $Q_{\text {low }}$ and $Q_{\text {hi }}$ with the $Q_{\text {mid }}$ homogeneous state forming an unstable separatrix. The spinodals mark the points of inflexion where $d^{2} g / d Q^{2}=0$. When the order parameter $Q$ lies within the spinodal boundaries, i.e., when $Q_{-}<Q<Q_{+}$, fluctuations about the initial state $Q_{\text {mid }}$ promote spontaneous precipitation into separated phases $Q_{\text {low }}$ and $Q_{\text {hi }}$. This is phase separation via spinodal decomposition.

The corresponding $Q-T$ phase diagram is presented in Fig. 3(b). The binary mixture is prepared in a hightemperature $\left(T=T_{2}>T_{c}\right)$ state within which the homogeneous phase $Q_{\text {mid }}$ is preferred and then abruptly quenched to a lower temperature $\left(T=T_{1}<T_{c}\right.$ ) lying inside the spinodal 


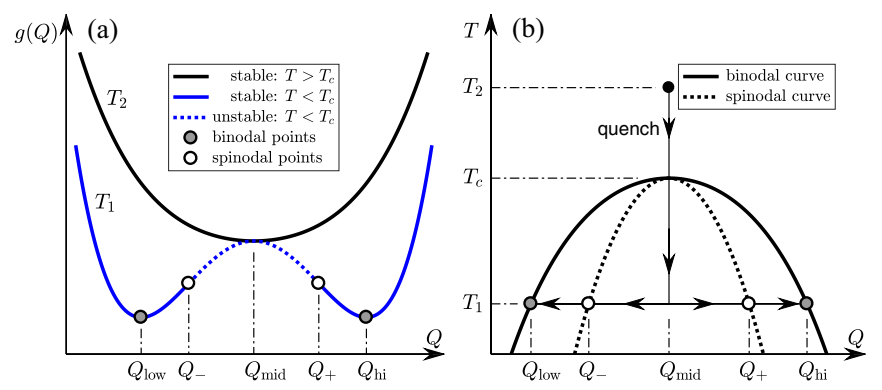

FIG. 3. Hypothetical free energy curves and phase diagram for an idealized "temperature"-driven spinodal decomposition of cortical activity. Here $Q$ represents the firing rate of the excitatory neural population with $0 \leqslant Q \leqslant Q_{e}^{\max }$. $T_{c}$ is the critical "temperature" below which homogeneous cortical activity $Q_{\text {mid }}$ spontaneously separates into high- and low-activity states $Q_{\mathrm{hi}}$ and $Q_{\text {low }}$, respectively. (a) Black upper free energy curve for elevated temperature $T_{2}>T_{c}$ shows a single minimum at activity $Q_{\text {mid }}$. Curve deforms downward into the blue (dark-gray) double-well potential at reduced temperature $T_{1}<T_{c}$ with minima corresponding to separated phases (binodal points) $Q_{\mathrm{hi}}$ and $Q_{\text {low }}$. Points of inflexion $Q_{-}, Q_{+}$mark the bounds of the unstable spinodal region. (b) Activity-temperature phase diagram showing a rapid temperature quench (vertical flow) from $T_{2}$ to $T_{1}$ inside the spinodal region, leading to separation into distinct phases (horizontal flows). (Panel B modified from Fig. 1 of Ref. [20].)

region. The homogeneous phase is now unstable with respect to small perturbations, so promptly decomposes into highand low-firing phases. If the initial $Q_{\text {mid }}$ homogeneous phase lies outside the spinodal limits but within the coexistence region $\left[Q_{\text {low }}, Q_{\mathrm{hi}}\right]$, then decomposition into nucleation cells can occur if there is sufficient system noise.

The theory for spinodal decomposition was developed $\sim 60$ years ago by Cahn, Hilliard, Hillert, and Cook [31-33]. Building from a free-energy formulation, these theoretical treatments showed that binary mixtures could separate into separated phases when the system is temperature quenched into the spinodal region.

For our eight-variable cortical model, it is not possible analytically to define a free energy. Instead we are guided by Cahn's approach [18] in which he linearizes the kinetic equations of the chemical system to derive an amplification factor $R$ that peaks at a critical wave number $q_{c}$. Cahn showed that, within the spinodal region, $R>0$, so wave-numberdependent fluctuations grow exponentially as $\exp \left[R\left(q_{c}\right) t\right]$, leading to development of a characteristic length scale with a superposition of sinusoidal waves of fixed wavelength but random orientations, phases, and amplitudes [34]. In fact, Cahn's amplification factor corresponds to the real part of the dominant eigenvalue in standard linear stability analysis: $R \equiv \alpha=\operatorname{Re}(\Lambda)$. This correspondence means that we can apply linear stability analysis to our cortical model as a means of predicting the emergence of spinodal decomposition. We need to acknowledge that these predictions are only valid in early-stage decomposition when fluctuations from the homogeneous mean are small.

But if spinodal decomposition is to be a plausible explanation for pattern formation in the cortex, then two questions immediately arise: (1) What is the cortical equivalent of thermodynamic temperature $T$ ? and (2) How does one impose a "thermodynamic quench" that "cools" the cortex through its critical point $T_{c}$ into the spinodal region?

As illustrated in Fig. 1, the equilibrium state of the cortex is determined by the codimensional interaction between excitation parameter $\Delta V_{e}^{\text {rest }}$ (resting-voltage offset for the excitatory population) and inhibition parameter $\lambda_{i}$ (scale factor for synaptic inhibitory gain). Tracing the edge of one of the two binodal coastlines, we see that an increase in excitation must be matched with a corresponding, albeit nonlinear, increase in inhibition (and vice versa). The two coastlines form a wedge that converges at the cusp $\mathrm{CP}$; at this point the distinction among activated, quiescent, and midbranch solutions disappears, and only the single equilibrium state remains. Therefore we take $\mathrm{CP}$ as marking the critical "temperature" $T_{c}$ and draw an imaginary line running approximately northwest to southeast down the central axis of the three-state wedge, passing through $\mathrm{CP}$, as our "temperature" axis, with effective temperature increasing with simultaneously larger values of $\Delta V_{e}^{\text {rest }}$ and $\lambda_{i}$.

A "thermodynamic quench" would place the cortex in the single-state region to the right and below CP and then rapidly "cool" the cortex along a northwest flow line, backward along the "temperature" axis, into a spinodal zone contained within the binodal coastlines. The noise-stimulated cortex would then phase separate via upward and downward vertical flows, settling onto the activated and quiescent equilibrium nodes. We characterize such a spinodal decomposition as nonconserved since the relative proportions of activated and quiescent cortex can take any value, unlike an alloy or chemical unmixing in which the number of atoms of either species is fixed.

Missing from Fig. 1 is any indication of the outer limits of the spinodal zone. This is because we cannot know the form of the free-energy function and therefore cannot locate its points of inflexion. Instead, we deduce the extent of the spinodal region via numerical simulations. While the spinodal explorations are preliminary at this stage, it appears that the spinodal zone for our cortical model is confined to a narrow strip just "inland" from the lower binodal coastline.

We now present the results from a suite set of spinodal investigations and comment on the time course and morphology of the resulting decomposition patterns. We note in advance that all stationary patterns observed so far are fundamentally hexagonal in structure.

\section{RESULTS}

\section{A. Spinodal decomposition simulations}

The numerical simulations reported in Secs. III A-III E were performed on a $240 \times 240$ grid with periodic (toroidal) boundaries representing a $25 \times 25-\mathrm{cm}$ continuum sheet of cortical tissue. In order to explore formation of finer-grain spinodal patterns, we also ran simulations on a $6 \times 6-\mathrm{cm}$ cortex at $240 \times 240$ grid sampling (Sec. III F). All grid points were continuously exposed to low-intensity spatiotemporal white noise. System equations were iterated in MATLAB using a forward-time, centered-space Euler-Maruyama scheme with time step $\Delta t=200 \times 10^{-6} \mathrm{~s}$ for the $25 \times 25$-cm cortex; smaller values for time step were tested but did not alter evolution dynamics or pattern properties. For the $6 \times 6-\mathrm{cm}$ cortex runs, $\Delta t$ was reduced to either $50 \times 10^{-6} \mathrm{~s}$ or $20 \times 10^{-6}$ 
$0.00 \mathrm{~s}$

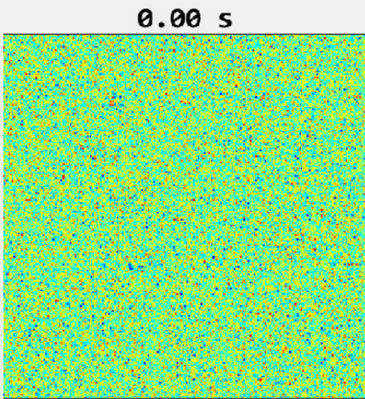

$0.36 \mathrm{~s}$

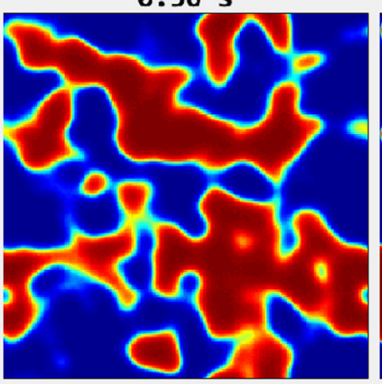

$0.20 \mathrm{~s}$

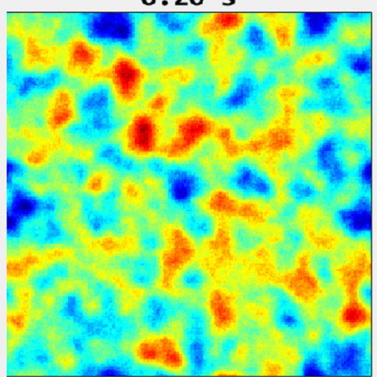

$1.00 \mathrm{~s}$

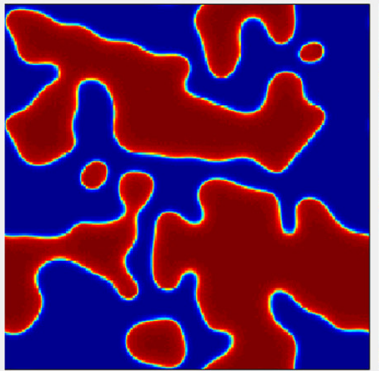

$0.26 \mathrm{~s}$

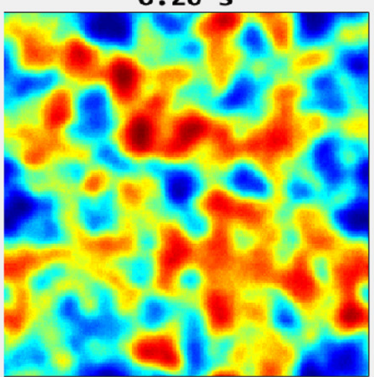

$5.00 \mathrm{~s}$

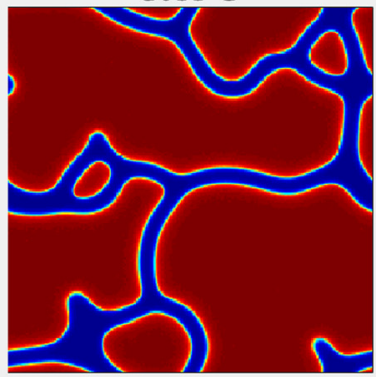

$0.30 \mathrm{~s}$

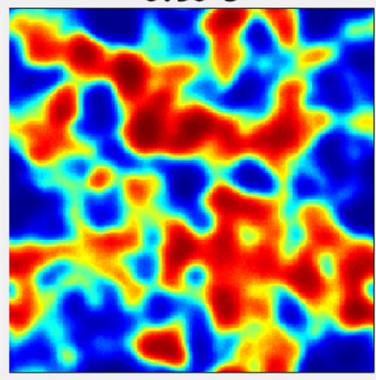

$60.00 \mathrm{~s}$

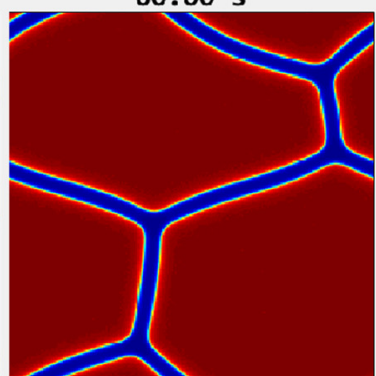

FIG. 4. Spinodal decomposition into a "honeycomb" structure: red (light gray with white borders) hexagonal islands of activity surrounded by narrow blue (dark-gray) canals of inactivity. Precipitation of jagged bulk structures occurs rather rapidly $(\sim 1 \mathrm{~s})$, and then the fine coastal details disappear as the pattern ripens on a much slower timescale (approximately tens of seconds). Parameter settings: $\left[\Delta V_{e}^{\text {rest }} / \mathrm{mV}, \lambda_{i}, D_{2} / \mathrm{cm}^{2}, \gamma_{i}^{0} / \mathrm{s}^{-1}\right]=[-1.85,0.7843,0.3,80]$; homogeneous model cortex was started on the midbranch equilibrium point for location (b) in Fig. 1. See Video 1 in the Supplemental Material [35] for a 60-s movie to accompany honeycomb structure emerging from homogeneous midbranch equilibrium.

s, depending on the value of inhibitory diffusion $D_{2}$ (see Sec. III F for details).

Simulations were initialized to the unstable midbranch equilibrium state separating the stable upper (activated) and stable lower (quiescent) equilibrium nodes for a given $(E, I)=\left(\Delta V_{e}^{\text {rest }}, \lambda_{i}\right)$ coordinate lying within the three-state region (Fig. 1) and allowed to run until the emergent patterns had fully matured. We found that only a small subset of the available three-state $(E, I)$ coordinates actually support stable pattern formation, and we presume that the pattern/nopattern boundary defines the areal extent of the spinodal zone lying within the broader binodal region. Selecting $(E, I)$ coordinates lying outside the spinodal zone would result in whole-of-cortex pattern collapse onto either the activated node or the quiescent node, depending on which of the two stable equilibria formed the stronger attractor.

We characterize the set of stable decomposition patterns as belonging to one of three broad classes: honeycomb, meander, and nucleation; these are described below. The time course for pattern evolution is discussed in Sec. III B. Evidence for a common hexagonal basis for cortical patterns is presented in Sec. III C. In Sec. IIID we demonstrate that stationary spinodal decompositions can be destabilized by proximity to a Hopf instability to form a shock wave that may relate to transient precursor electrical activity observed prior to seizure onset in cortical tissue. We examine the range of spinodal shapes accessible to the cortical model in a series of 18 "prospecting" experiments distributed along the binodal coastline in Sec. III E, and in Sec. III F we conclude Sec. III by exploring the conditions under which fine-scale (of order $\sim \mathrm{mm}$ ) spinodal activity patterns can be generated.

\section{Honeycomb}

The 2D cortical sheet was initialized to the unstable midbranch equilibrium associated with coordinate (b) in the threestate region of Fig. $1,\left(\Delta V_{e}^{\text {rest }} / \mathrm{mV}, \lambda_{i}\right)=(-1.85,0.7843)$, and then allowed to evolve under the influence of continuous low-intensity spatiotemporal white noise. Clusters of high and low activity promptly emerge from the homogeneous initial state $(t=0.2 \mathrm{~s})$ and then consolidate into islands of activity (red) surrounded by a sea of quiescence (blue); see Fig. 4 $(t=1 \mathrm{~s})$. The activity islands then slowly enlarge over a period of seconds, gradually compressing the inactive zones into narrow canals $(t=5 \mathrm{~s})$ before finally reshaping into large hexagonal continents $(t=60 \mathrm{~s})$.

\section{Meander}

The meander simulation of Fig. 5 is located at coordinate (a) in Fig. 1, closer to the three-state/one-state cusp marking the critical point CP. As was the case for honeycomb, the bulk patterns are already clear at $t=1 \mathrm{~s}$, with the subsequent gradual ripening to maturity occurring over tens of seconds. However, the pattern structure is now highly irregular: a mazelike labyrinth of activity channels isolated by meanders of inactivity. While this spatial structure is strongly reminiscent of the Turing patterns reported in earlier work [13], Fig. 5 is the result of spinodal decomposition rather than a Turing instability. This is made clear in the stability curves of Fig. 2(a); although the bottom branch dispersion curve shows a Turing-like peak, it is damped, and both top and bottom branches are stable attractors rather than sources of instability. 

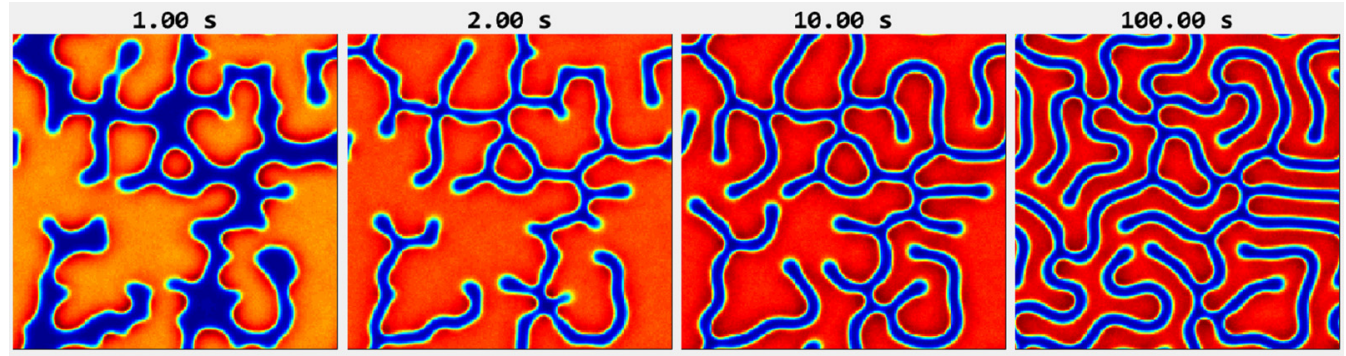

FIG. 5. Spinodal decomposition into a "meander" structure: a mazelike labyrinth of continuously linked blue (black) canals of quiescence isolating red (white-bordered gray) islets of activity. As for the honeycomb of Fig. 4, the bulk pattern is already apparent after $\sim 1 \mathrm{~s}$ and then matures more slowly. Settings: $\left[\Delta V_{e}^{\text {rest }} / \mathrm{mV}, \lambda_{i}, D_{2} / \mathrm{cm}^{2}, \gamma_{i}^{0} / \mathrm{s}^{-1}\right]=[1.3,1.0,0.35,80]$; initial state: midbranch steady state for location(a) in Fig. 1. See Video 2 in the Supplemental Material [35] for a 100-s movie to accompany emergence of a Turing-like labyrinthine cortical activation.

\section{Nucleation and mitosis}

The nucleation simulation is located at coordinate (c) in Fig. 1 in a region of weakened $(E, I)$ drive and reported in Fig. 6. As for honeycomb and meander, bulk patterns have emerged at $t=1 \mathrm{~s}$, but subsequent evolution is very different. Here larger islands of activity pinch off and separate into daughter cells, while smaller nuclei grow until they reach a critical size, thin and elongate, and then split into pairs of daughter cells. Population growth by mitosis continues until crowding pressures suppress the expansion.

\section{Effect of noise intensity on pattern emergence and stability}

All of the numerical simulations reported here are driven by low-intensity spatiotemporal white noise; see Eq. (4). Previous modelers investigating phase separation dynamics have reported that the inclusion of noise in spinodal simulations is essential to prevent artificial quenching at local minima of the free-energy functional [36]. This motivated us to probe the role of noise in pattern emergence and early dynamics for our cortical model.

We repeated the nucleation experiments reported in Fig. 6 for a wide range of settings for $s$, the amplitude of the spatiotemporal white noise; see Fig. 7. These numerical experiments revealed three general properties:

(i) if the noise amplitude is zero, then nucleation patterns never form; (ii) provided the noise is non-negligible, pattern morphology is both robust and remarkably insensitive to noise amplitude;

(iii) pattern emergence and maturation is hastened by larger-amplitude noise.

Our simulations commence on the unstable midbranch separatrix. Because this is an equilibrium point, the rates of change of all state variables are-by definitionsimultaneously zero, so if the cortical equations are unperturbed by noise, then the systems will be frozen there "forever" [or, more precisely, until numerical errors in the double-precision (64-bit) floating-point arithmetic accumulate sufficiently to form an artificial noise disturbance]. Writing the noise scaling as $s=k s_{0}$ (with $s_{0}=0.125$ being the default value), we found that if the amplitude is set too small $\left(k \lesssim 10^{-10}\right)$, then patterns fail to emerge. However, for the range $10^{-8}<k<5$ (i.e., $\sim 8.5$ orders of magnitude), nucleation patterns emerged promptly and reliably, with the rate of pattern emergence and maturation being (weakly) accelerated by larger amplitude noise. Note that these dynamical properties are universal across the range of spinodal patterns reported here and are not specific to nucleation.

But if the noise is too intense, then patterns are eventually lost in the noise. This is apparent in the fourth column of Fig. 7 $(k=80)$.
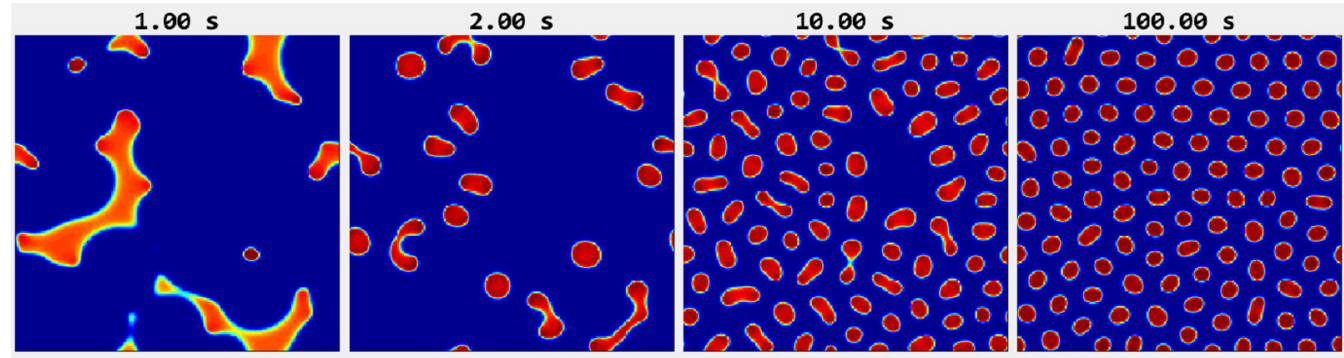

FIG. 6. Spinodal decomposition into an almost regular "nucleation" pattern: a cellular array of red (white-bordered gray) activity nuclei in a sea of blue (black) quiescence. Larger structures pinch off and separate into daughter cells; smaller structures grow until they become unstable, whereupon they elongate into a dumbbell shape that thins and divides into pairs of daughter cells in a process reminiscent of biological mitosis. Cell population multiplies until the evident repulsive force between nearest neighbors suppresses the tendency of each cell to grow and divide. Settings: $\left[\Delta V_{e}^{\text {rest }} / \mathrm{mV}, \lambda_{i}, D_{2} / \mathrm{cm}^{2}, \gamma_{i}^{0} / \mathrm{s}^{-1}\right]=[-2.5,0.8,0.45,45]$; initial state: midbranch steady state for location-(c) in Fig. 1. See Video 3 in the Supplemental Material [35] for a 60-s movie to accompany emergence of an array of activity cells undergoing mitosis. 


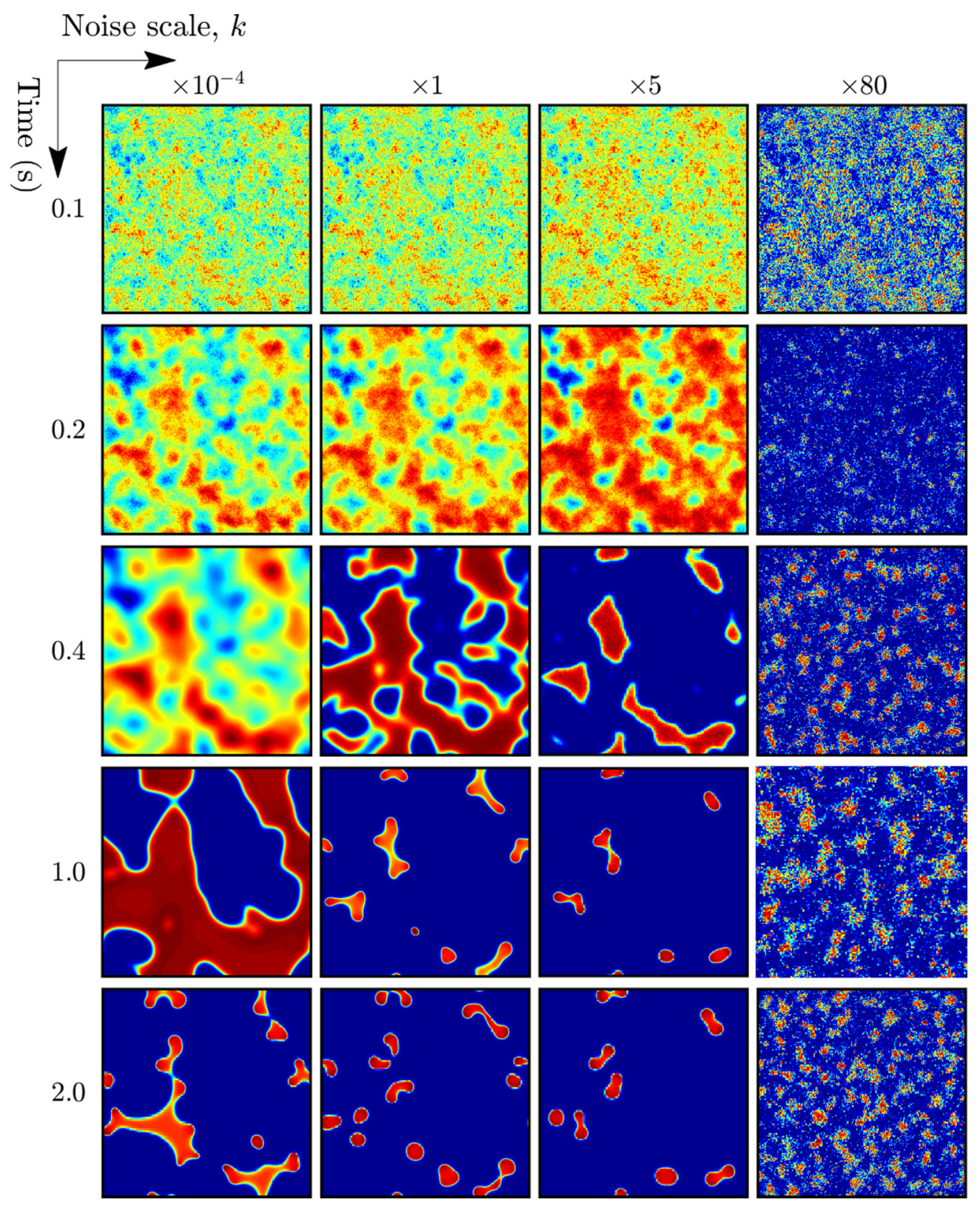

FIG. 7. Impact of noise intensity on emergence of nucleation spinodal patterns. Each column corresponds to one of four simulation experiments $k=\left[10^{-4}, 1,5,80\right]$, where $k$ scales the amplitude of the default setting for white-noise coefficient $s_{0}=0.125$ [see Eq. (4) and Table I]; time increases vertically downward. Each simulation uses the identical sequence of random numbers. In general, stronger stochastic stimulation promotes faster nucleation, but patterns became unstable if the noise is too intense (fourth column: $k=80$ ).

\section{B. Three phases of spinodal decomposition in the cortex}

In general, we find that patterns generated by spinodal decomposition in the cortex typically emerge very rapidly and then mature into final form much more slowly. This slow phase is described in the metallurgy literature as coarsening or Ostwald ripening. In fact, examination of the spatial spectra of the various cortical quench patterns reveals three distinct dynamical regimes that we label precipitation, differentiation, and maturation; these phases of development are illustrated in Fig. 8 for the formation of the cellular nucleation pattern of Fig. 6. We track the changes in the proportion of highand low-wave-number content, relative to a selected midrange reference wave number $\left(q^{\text {ref }} / 2 \pi=0.38 \mathrm{~cm}^{-1}\right)$, as a function of time. We define the high- and low-wave-number fractions across the cortical grid as

$$
\begin{aligned}
& f_{\mathrm{hi}}(t)=\frac{1}{\mathcal{N}(t)} \int_{q^{\mathrm{ref}}}^{q_{y}^{\mathrm{max}}} \int_{q^{\mathrm{ref}}}^{q_{x}^{\mathrm{max}}} \widetilde{Q}_{e}\left(q_{x}, q_{y} ; t\right) d q_{x} d q_{y}, \\
& f_{\mathrm{lo}}(t)=\frac{1}{\mathcal{N}(t)} \int_{0}^{q^{\mathrm{ref}}} \int_{0}^{q^{\mathrm{ref}}} \widetilde{Q}_{e}\left(q_{x}, q_{y} ; t\right) d q_{x} d q_{y},
\end{aligned}
$$

where $\widetilde{Q}_{e}\left(\vec{q}=q_{x}, q_{y} ; t\right)$ is the zero-mean spectral amplitude at time $t$ computed from the 2D spatial Fourier transform of the grid of excitatory firing rates $Q_{e}(\vec{r}=x, y ; t)$

$$
\widetilde{Q}_{e}(\vec{q}, t)=\left|\mathcal{F}\left[Q_{e}(\vec{r}, t)-\overline{Q_{e}(\vec{r}, t)}\right]\right|
$$




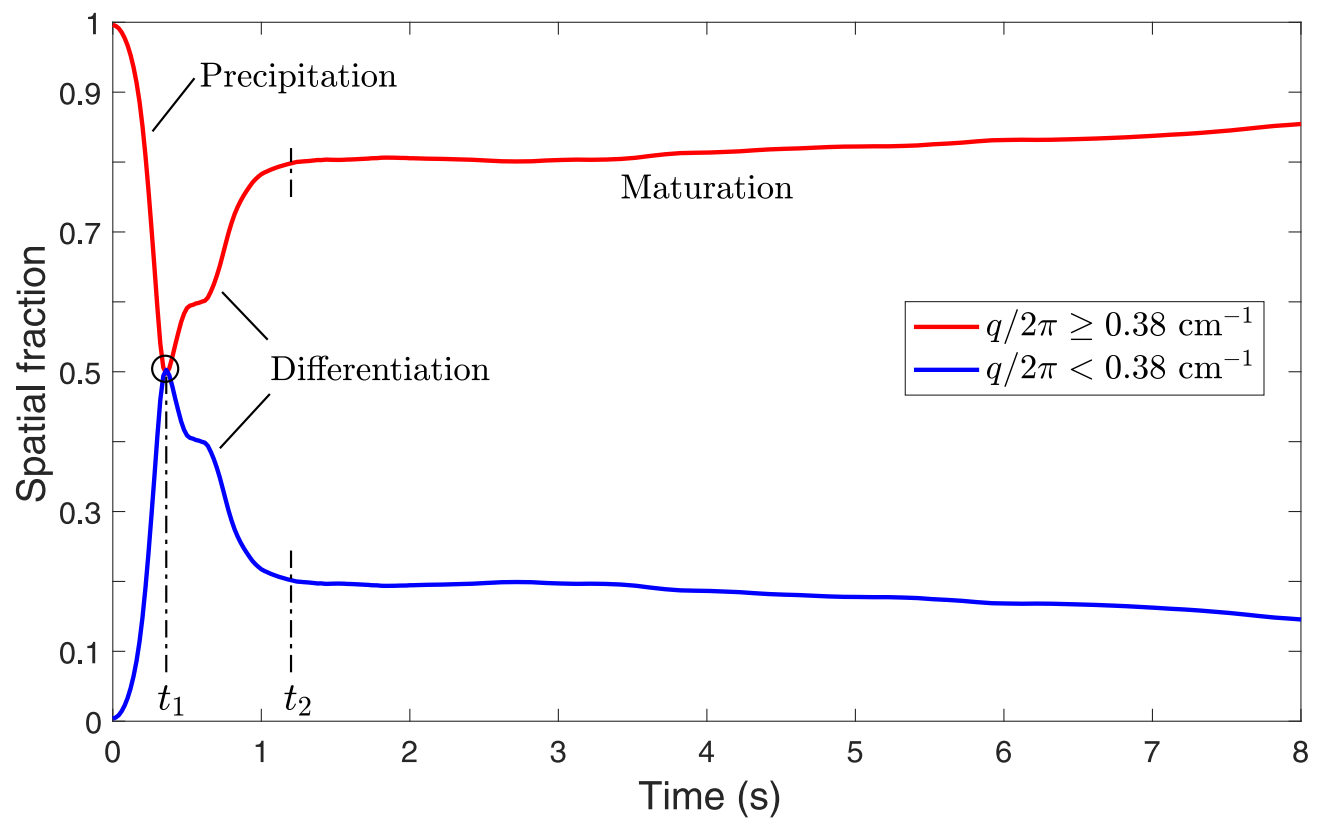

FIG. 8. Time development of the cellular nucleation pattern of Fig. 6 expressed as relative proportions of spectral content above and below a reference wave number, $q / 2 \pi=0.38 \mathrm{~cm}^{-1}$. At $t=0$, the unstable midbranch of the homogeneous model cortex is perturbed with small-amplitude spatial white noise. The upper red (gray) curve shows that the initially high spatial-frequency fraction falls precipitously as large amorphous structures emerge spontaneously to break symmetry, reaching a minimum at $t_{1} \approx 0.4 \mathrm{~s}$. The lower blue (black) curve tracks the low-frequency spatial energy which peaks at this point, signaling the onset of a structural phase transition. The high-wave-number fraction then grows rapidly as the differentiation into high- and low-firing cortical patches proceeds and then matures more slowly after $t_{2} \approx 1.25 \mathrm{~s}$.

with $\overline{Q_{e}(\vec{r}, t)}$ being the average firing rate across the grid at time $t$. Here $\mathcal{N}(t)$ is a normalization factor that fixes the total volume under the $2 \mathrm{D}$ spectral surface at unity, and thus $f_{\mathrm{hi}}(t)+f_{\mathrm{lo}}(t)=1$.

In Fig. 8, precipitation describes the fast transformation from a fine-grained white-noise perturbed homogeneous quench state $\left(f_{\text {hi }} \approx 1, f_{\text {lo }} \approx 0\right)$ to primordial amorphous clouds of high- and low-firing neural populations ( $f_{\mathrm{hi}}$ subsides as $f_{\text {lo }}$ approaches its peak). There is no hint of the final structure at this primeval stage. This is followed by an equally rapid phase transformation in which the amorphous shapes promptly differentiate into structured elements, causing $f_{\mathrm{hi}}$ to increase steeply, and then more slowly as the maturation phase begins. This monotonic increase in $f_{\text {hi }}$ is consistent with the fact that the matured cellular pattern forms a hexagonal latticework (see top row of Fig. 9) with characteristic wave number $\left(0.44 \mathrm{~cm}^{-1}\right)$.

\section{Underlying hexagonal basis of decomposition patterns}

Despite the disparate appearances of the mature honeycomb (Fig. 4), meander (Fig. 5), and nucleation (Fig. 6) spinodal decomposition patterns, inspection of their spatial spectra suggests an underlying hexagonal organization. The 2D spatial Fourier transforms of the nucleation and meander patterns in Figs. 9(b) and 9(e) show an annular ring structure with dominant wave number $q / 2 \pi \approx 0.4$ waves $/ \mathrm{cm}$, corresponding to the $\sim 2.5-\mathrm{cm}$ repetition length in the cell and maze patterns in Figs. 9(a) and 9(d). As evident in Figs. 9(d) and $9(\mathrm{e})$, these ring structures become more clearly defined when averaged over 25 spectral instances. The fact that the spectra do not exhibit discrete intensity peaks is consistent with the decomposition patterns having pattern defects and orientation irregularities and so look more like the scattering pattern of a powdered crystal with random orientations rather than that of a regular crystal. The structure defects are clearest in the nucleation pattern of Fig. 9(a): Cell shapes vary between circular and elongated and form a lattice that is primarily hexagonal but contains some five- and seven-nearest-neighbor groupings.

\section{Soliton generation from spinodal-Hopf interaction}

The honeycomb, meander, and nucleation patterns all exhibit the three-stage (precipitation, differentiation, maturation) evolution dynamics illustrated in Fig. 8, with spatial rates of change gradually relaxing to zero over time. This is expected behavior for spinodal decomposition into stationary patterns attracted to the stable binodes defined by the top- and bottom-branch equilibria.

In previous explorations of our cortical model [14,37-39], we have demonstrated that interactions between spatial (Turing) and temporal (Hopf) instabilities can lead to a range of oscillating, traveling, and chaotic spatiotemporal patterns, with strong Hopf dominance leading to suppression of chaos in favor of slow, coherent traveling waves that invade the entire cortex and which may correspond to epileptic seizure [39]. This earlier work motivates us to ask: What happens when a spinodal decomposition process interacts with weakly damped temporal instability?

Using the same $(E, I)$ settings as for the honeycomb simulation (Fig. 4), we lowered the inhibitory rate constant by a factor of $\sim 4\left(\gamma_{i}=80 \rightarrow 22 \mathrm{~s}^{-1}\right)$ to bring the top-branch dispersion curve of Fig. 2(d) very close to a zero-wave-number 

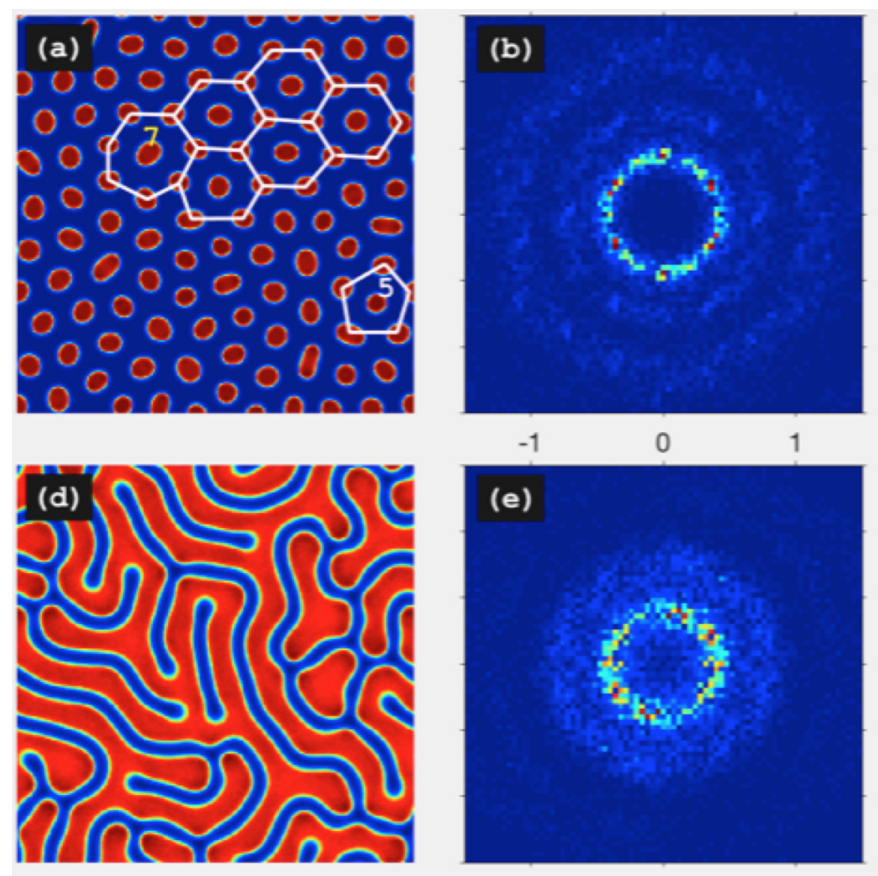

0

1

$-1$

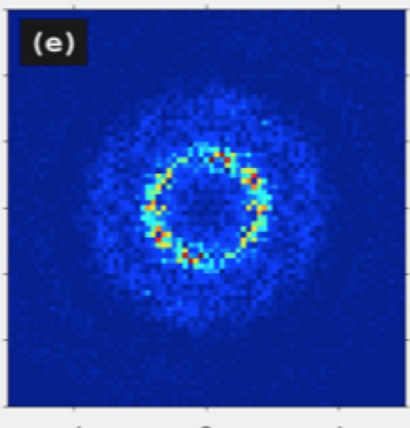

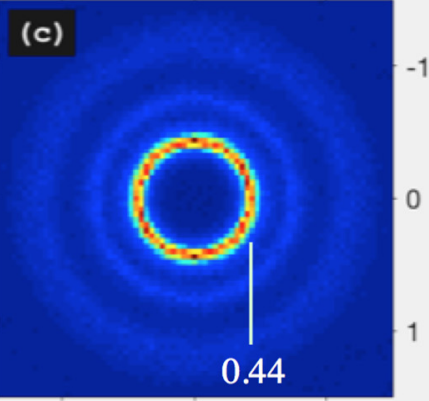

0

1

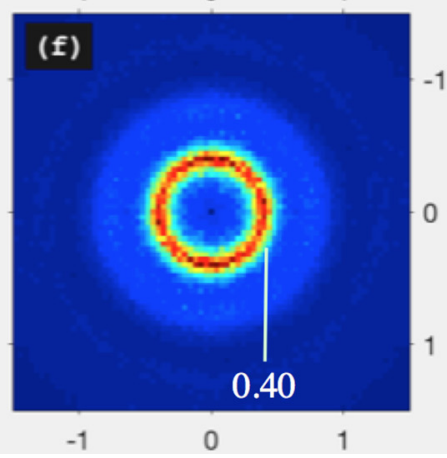

FIG. 9. Hexagonal structuring of (a) cellular and (d) labyrinthine decomposition patterns revealed by 2D spatial Fourier transforms in panels (b) and (c), respectively; panels (c) and (f) show the amplitude spectra obtained after averaging over 25 sets of 100-s grid simulations. The radius of the primary ring structures $\left(q / 2 \pi=0.44\right.$ and $\left.0.40 \mathrm{~cm}^{-1}\right)$ correspond to the inverse of the characteristic repetition lengths of the cell and maze patterns of (a) and (d). In (a), some representative cells have been joined (white lines) to aid the eye; while most show the expected six-nearest-neighbor hexagonal links, structure defects are evident with some seven- and five-neighbor arrangements.

instability such that the top branch is still linearly stable but only marginally so (dominant eigenvalue has real part $\alpha=-0.001 \mathrm{~s}^{-1}$ at $\left.q=0\right)$.

Simulation results are shown in Fig. 10. We find a qualitatively different and new dynamics in which the initial decomposition results in vigorous competition between activated and quiescent patches; these interactions eventually condense onto a narrow soliton-like shock-front of activity that traverses the cortex at $\sim 4 \mathrm{~cm} / \mathrm{s}$. Since this near-Hopf setting produces soliton waves, and a full-Hopf instability produces putative seizure, we argue that these soliton fronts may correspond the the interictal waves of population-spike activity commonly seen between periods of full seizure.

Figure 11 shows the time course of a typical seizurelike event recorded from five electrodes placed in a slice of mouse cortex. We estimate the propagation speed at $3-5 \mathrm{~cm} / \mathrm{s}$, providing a pleasing match with the soliton speed in the simulation, and with the published $10 \pm 9 \mathrm{~cm} / \mathrm{s}$ range of speeds for interictal spikes detected in tissue slices from human subjects [40,41]. Unlike the soliton wavelet in our homogeneous spinodal model, the wave of electrical activity in the mouse brain changes shape and attenuates as it moves, presumably because the biological tissue is neither isotropic nor homogeneous.

\section{E. Diversity of spinodal patterns}

In order to survey of the types of spinodal decomposition patterns accessible to the mean-field cortical model, we ran a series of simulations for a range of $(E, I)$ coordinates distributed across the binodal region. Most of these prospecting simulations resulted in eventual homogeneous collapse onto either the activated (high-firing) or quiescent (low-firing) stable branches (not shown). However, we found a narrow strip of coordinates-just inland from the lower binodal coastline marking the three-state/one-state boundary-that supported stable patterns. The type of pattern could be controlled both by small displacements toward or away from the coast and by displacements parallel to the coast.

Figure 12 shows the results of 18 prospecting simulations, traveling southeast along the coast in the direction of increasing $(E, I)$ drive. Each numerical experiment was halted after $10 \mathrm{~s}$, so the captured patterns are precursive indicators of the fully matured decompositions. As $(E, I)$ drive increases, the patterns evolve in a general sequence: (honeycomb precursor) $\rightarrow$ (cellular nucleations: active cells in a background of quiescence) $\rightarrow$ (meandering labyrinths), eventually terminating in inverse-nucleation patterns (cells of quiescence embedded in a sea of activity) as we approach the cusp marking the critical point. In all stable-pattern cases, the fully matured decompositions share a fundamentally hexagonal spatial structure.

\section{F. Length scale of spinodal patterns}

Many of the spinodal patterns exhibited here have spatial extents covering several centimeters, so are probably too large to be biologically plausible, particularly given our assumption of a homogeneous isostropic cortex. This has motivated us to investigate the conditions under which millimeter-scale patterns might emerge.

Of the $\sim 27$ model parameters listed in Table I, only a small subset $\left(D_{1,2}, \gamma_{e, i}, \Lambda_{e b}, \tau_{e, i}, v\right)$-i.e., diffusions, rate 
$0.02 \mathrm{~s}$

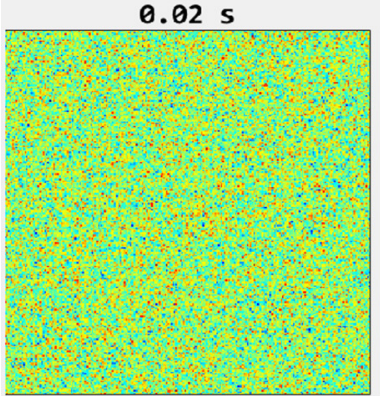

$2.00 \mathrm{~s}$

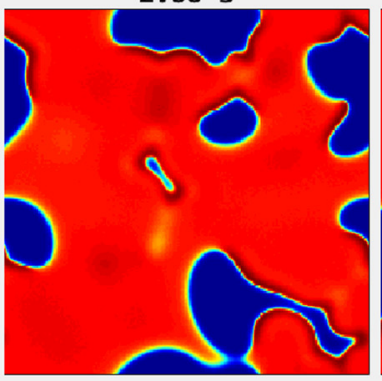

$0.20 \mathrm{~s}$

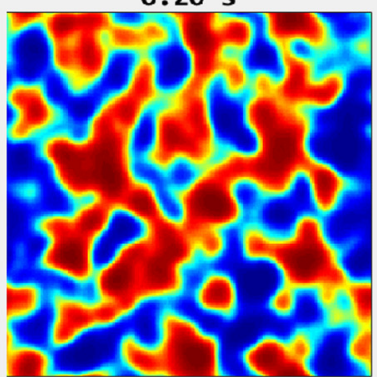

$5.00 \mathrm{~s}$

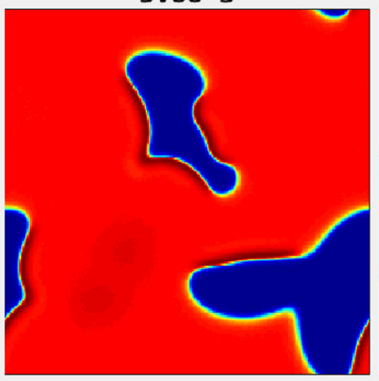

$0.40 \mathrm{~s}$

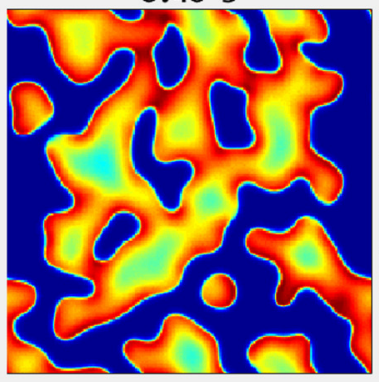

$26.00 \mathrm{~s}$

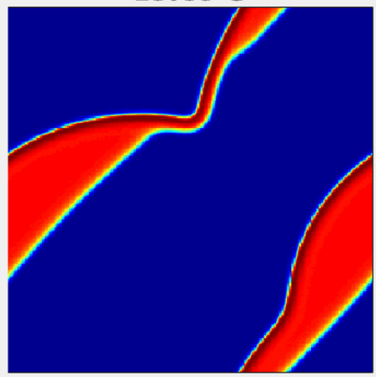

$1.00 \mathrm{~s}$

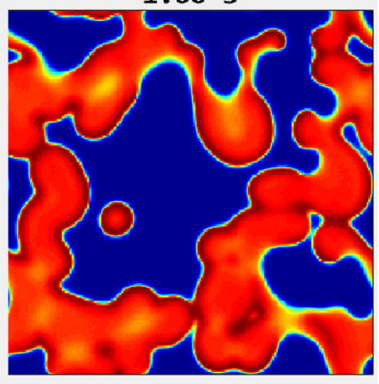

$30.00 \mathrm{~s}$

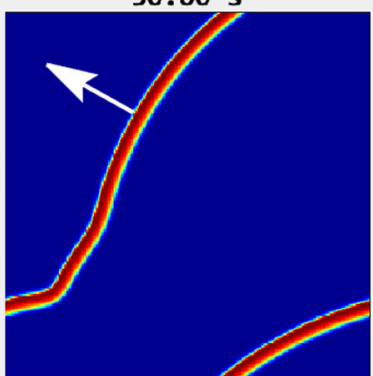

FIG. 10. Destabilization of spinodal phase separation by close proximity to a Hopf bifurcation. Cortex was initialized at the same $\left(\Delta V_{e}^{\text {rest }}, \lambda_{i}\right)$ starting coordinate that produced the stationary honeycomb pattern of Fig. 4, but the inhibitory rate-constant was reduced by a factor of $\sim 4$ to induce a marginally damped Hopf instability in the upper branch (see Fig. 2(d)). Although initial coastline shapes are similar (compare the 0.2-s panel here with the 0.3-s panel of Fig. 4), temporal evolution is now qualitatively different with vigorous competition between red (white-bordered gray) activated and blue (black) quiescent patches that eventually resolves into a soliton-like wave of activity that traverses the cortex at $\sim 4 \mathrm{~cm} / \mathrm{s}$. See Video 4 in the Supplemental Material [35] for a 38-s movie to accompany emergence of a soliton

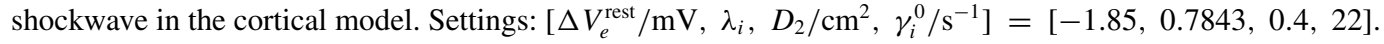

constants, time constants, and the like-can be varied without disturbing cortical steady state. Since proximity to the multiroot region is essential for spinodal pattern formation, it is essential not to lose contact with the binodal coastlines in these parameter-space explorations. For this reason we chose not to alter synaptic gains, synaptic connection counts, or sigmoidal voltage-to-firing-rate transfer functions, since changes in any one of these parameters will alter the underlying distribution of cortical steady states.

We selected the nucleation coordinate (c) in Fig. 1 (see Fig. 6 caption for settings) as the reference point for our search for finer-grained spinodal patterns. We found that pattern granularity (i.e., wave-number spatial frequency) could be increased by reducing inhibitory diffusion $D_{2}$ simultaneously with increases in $\Lambda_{e b}$, the excitatory axonal spatial decay rate. Because the fine-grained patterns eventually become impossible to resolve at $240 \times 240$ resolution on the default $25 \times 25-\mathrm{cm}$ grid (i.e., $1.04-\mathrm{mm}$ pixels), we down-scaled the cortex to a $6 \times 6-\mathrm{cm}$ grid at $240 \times 240$ to give $0.25-\mathrm{mm}$ pixels. In order to guarantee numerical stability, this change in spatial resolution necessitated a reduction in simulation time step from the default value of $\Delta t=200 \times 10^{-6} \mathrm{~s}$ on the $25-\mathrm{cm}$ cortex to $50 \times 10^{-6} \mathrm{~s}$ for $D_{2}<0.20 \mathrm{~cm}^{2}$ and $20 \times 10^{-6} \mathrm{~s}$ for $D_{2} \geqslant 0.20 \mathrm{~cm}^{2}$. Thus simulations on the smaller fine-grained
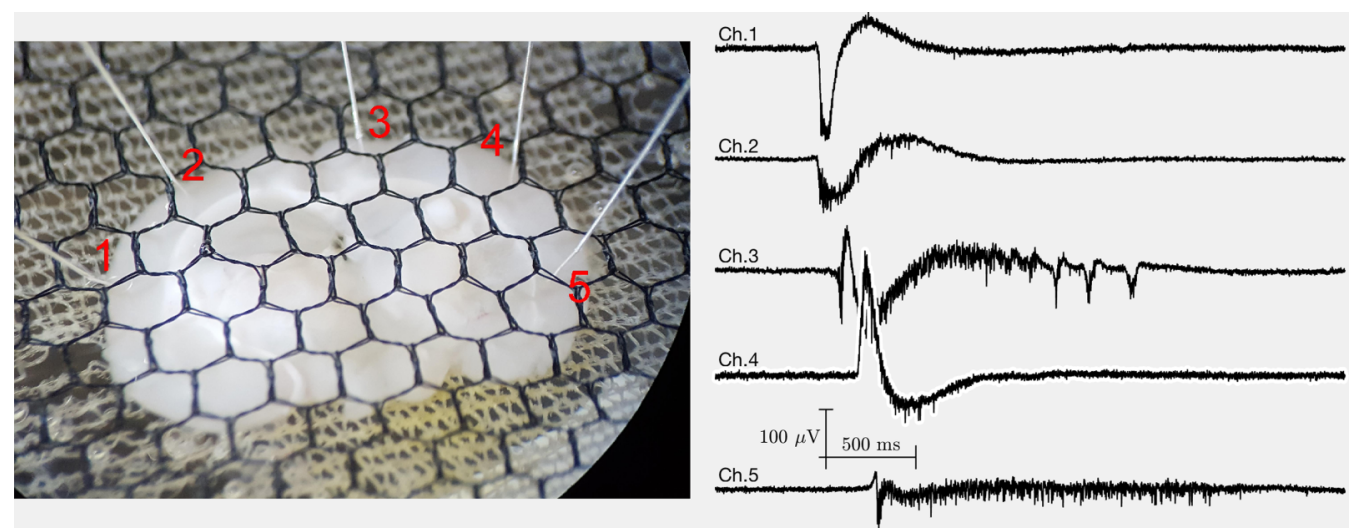

FIG. 11. Time series for a seizurelike precursor event in mouse cortex. The electrical activity of a 400- $\mu$ m-thick coronal slice of mouse brain tissue is sampled with five numbered $\mathrm{Ag} / \mathrm{AgCl}$ electrodes (left photo). A seizurelike event (SLE) impinges simultaneously on electrodes 1 and 2 (right-hand traces) and then traverses the cortical rind to reach electrodes 3, 4, and 5 at successively later times. The mesh spacing is $\sim 1.5 \mathrm{~mm}$, giving an SLE travel speed of 3 to $5 \mathrm{~cm} / \mathrm{s}$. [See the Appendix for experiment details.] 

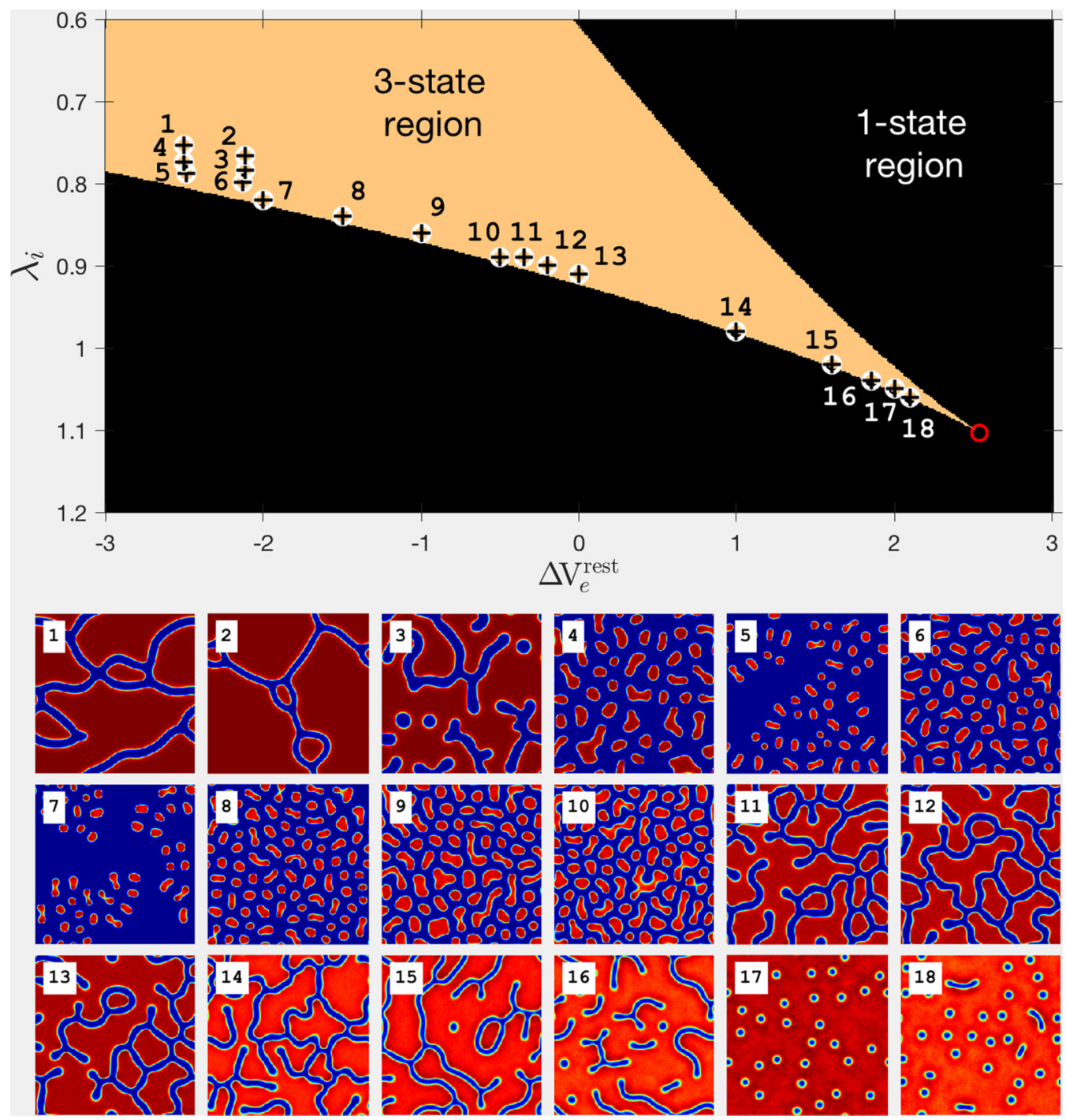

FIG. 12. Gallery of representative spinodal decomposition patterns for $18\left(\Delta V_{e}^{\text {rest }}, \lambda_{i}\right)$ coordinates located slightly inland from the lower binodal coastline. Each snapshot shows early pattern development $10 \mathrm{~s}$ after launching from the unstable midbranch separatrix. In addition to precursor honeycomb (panels 1-3), red-on-blue (gray-on-black) nucleation (4-10), and meander (11-16) patterns, blue-on-red (black-on-gray) inverse nucleations are also evident (panels 17 and 18 ). Spatiotemporal settings: $\left[D_{2} / \mathrm{cm}^{2}, \gamma_{i}^{0} / \mathrm{s}^{-1}\right]=[0.35,80]$.

cortical grid ran $\sim 3-10$ times slower than on the larger coarse grid.

Four representative results for the small-cortex simulations are displayed in the top row of Fig. 13; the bottom row shows the corresponding spatial spectra. As expected, the more finely structured labyrinthine and nucleation patterns (viewing left-to-right along top row) exhibit successively higher dominant wave numbers with spatial fluctuation energy peaking at larger annular radius (bottom row). Figure 13(d) shows a strong spectral peak at $q / 2 \pi \approx 2.67 \mathrm{~cm}^{-1}$ corresponding to a pattern periodicity of $\sim 3.7 \mathrm{~mm}$; this is about six times smaller than the $\sim 23-\mathrm{mm}$ spatial scale of the default nucleation pattern of Fig. 6.

Figure 14(a) illustrates how the spectral peak for the Fourier spectrum of Fig. 13(d) is determined by computing the radially averaged spatial activity and then smoothing with a Whittaker filter [42]. Figure 14(b) shows the approximately linear trend obtained when the dominant wave number at each of 20 simulations (13 runs at $L_{x, y}=25 \mathrm{~cm}$, plus 7 runs at $6 \mathrm{~cm}$ ) is plotted versus the composite ratio $0.183\left[\Lambda_{e b} / \sqrt{D_{2}}\right]^{1 / 2}$. This proportionality follows from our empirical discovery that

$$
q_{\mathrm{dom}}^{2} \sim \Lambda_{e b} / \sqrt{D_{2}}
$$

where $\Lambda_{e b}^{-1}$ is the length scale for excitatory axonal connections and $\sqrt{D 2}$ is an effective "side-length" for inhibitory gapjunction diffusive connections. Thus finer spinodal structures are associated with smaller axonal ranges (i.e., larger values of $\Lambda_{e b}$ ) and smaller values of inhibitory diffusion.

\section{DISCUSSION}

In this paper we propose spinodal decomposition as a novel agent for hexagonal pattern formation in the cortex. Standing in marked contrast to conventional pattern-formation models based on network-attractor theory [e.g., 43-45], spinodal decomposition supports spontaneous emergence of patterns 

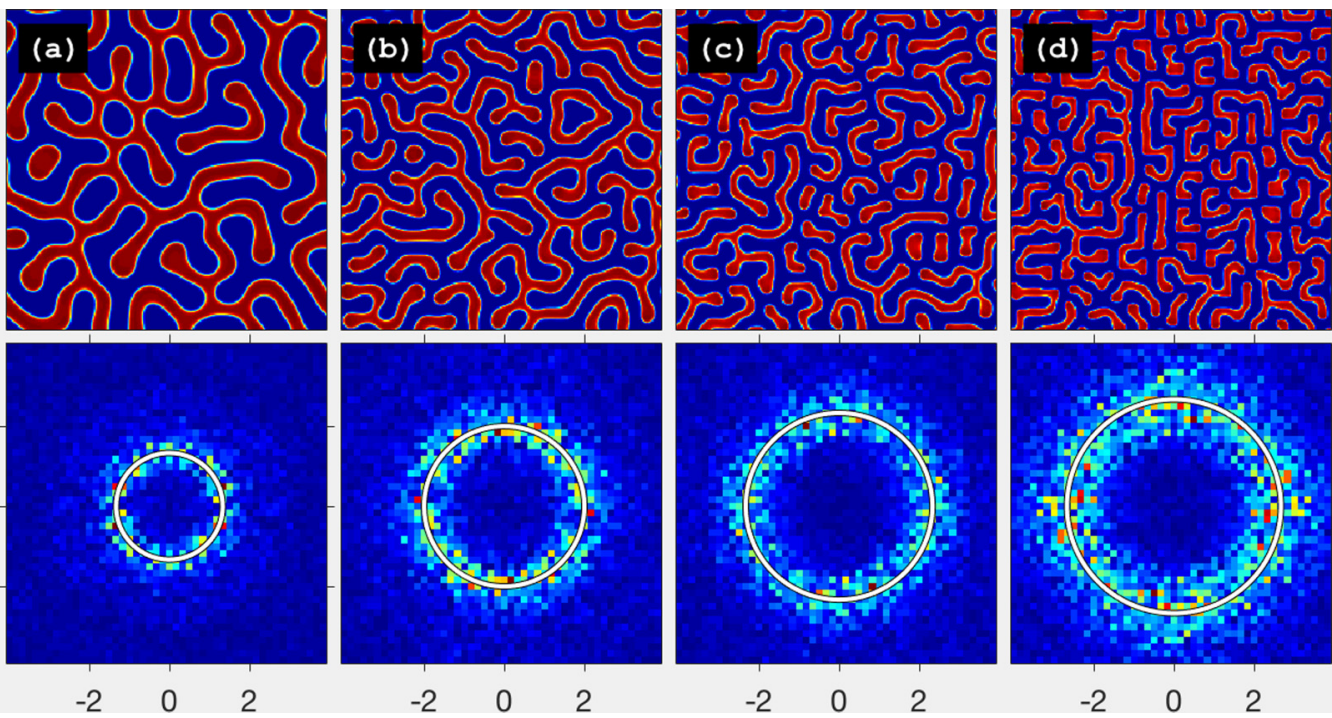

FIG. 13. Spinodal patterns (first row) and spatial spectra (second row) developed after $10 \mathrm{~s}$ on a $6 \times 6$-cm cortex. From left to right, respective inhibitory diffusion $\left(D_{2}\right)$ and excitatory axonal decay rate $\left(\Lambda_{e b}\right)$ are $D_{2} / \mathrm{cm}^{2}=[0.20,0.05,0.02,0.015]$ and $\Lambda_{e b} / \mathrm{cm}^{-1}=$ [24, 24, 24, 28]. Other model settings are identical to those used for the nucleation patterns of Figs. 1 and 6 on a $25 \times 25-\mathrm{cm}$ cortex. [(a)-(d)] As diffusive and axonal connectivities reduce, the characteristic length scales of the spinodal patterns shrink, and, consequently, the dominant spatial mode (second row) increases in frequency (units: $\mathrm{cm}^{-1}$ ). White ring indicates dominant spatial frequency obtained from radial averaging [see Fig. 14(a)].

of high- and low-firing activity without requiring learning rules or growth laws to tune synaptic strengths and without imposing an inhibitory annular surround as seen in Mexican hat (and related) lateral-inhibition connectivity kernels.

Our spinodal model requires access to a pair of stable equilibrium nodes, isolated by an unstable separatrix, and a modest level of inhibitory gap-junction diffusion $\left[D_{i i}=D_{2}\right.$ in Eq. (1)] that is insufficient to destabilize either node (see growth-rate curves in the upper and lower panels of Fig. 2). This latter requirement distinguishes the present work from previous investigations of our cortical model [13,14,37-39] in which strong inhibitory diffusion leads to destabilization of a previously stable node via Turing bifurcation.

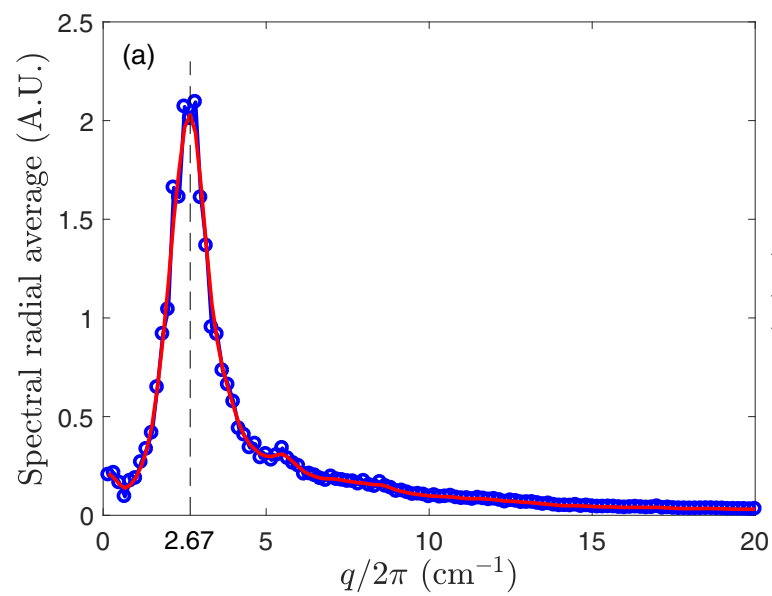

It is clear that the fully developed spinodal patterns reported here (e.g., Figs 1 and 9) have a fundamentally hexagonal basis. At low values of $(E, I)$ drive [coordinate (c) in Fig. 1], we see spontaneous nucleation: formation and mitotic growth of "activity cells" that evolve into a quasistationary hexagonal array (Fig. 6).

The prospect of hexagonal spatial patterning in the brain, and its potential association with representation and memory, has been vigorously debated since the late 1950s. Quasihexagonal patterns have been observed in the clustering of cortical minicolumns [46-48]: vertical columns extending through the layers of the cortex, containing 100-200 neurons; Mountcastle (1957) argues that minicolumns are the

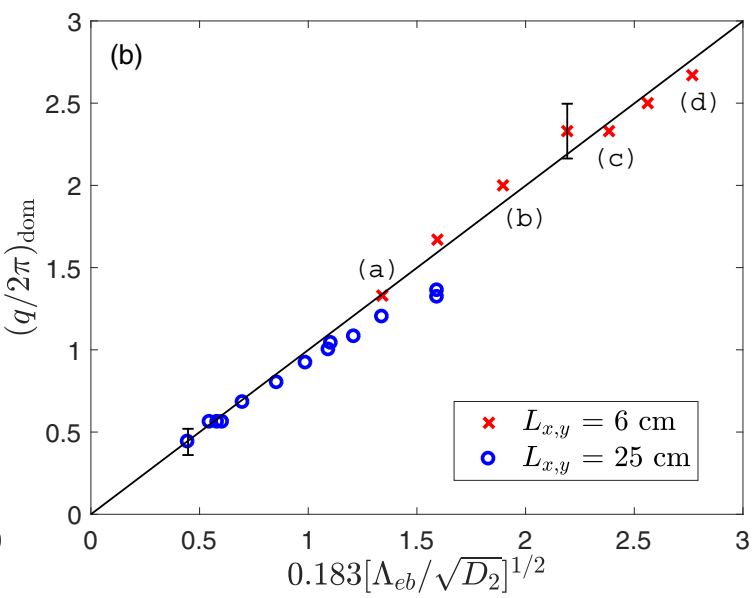

FIG. 14. (a) Radially averaged spatial activity for spinodal pattern Fig. 13(d). (b) Wave-number trend with axonal decay-rate and inhibitory diffusion (right) across 20 spinodal simulations and two cortical domain sizes. Evidently the dominant wave number follows a linear trend $q_{\mathrm{dom}} \propto\left[\Lambda_{e b} / \sqrt{D_{2}}\right]^{1 / 2}$ across a sixfold change in characteristic length scale from $1 / 0.44 \mathrm{~cm}^{-1}=2.3 \mathrm{~cm}\left(\right.$ lower-left datum) to $1 / 2.67 \mathrm{~cm}{ }^{-1}=$ $0.37 \mathrm{~cm}$ (upper-right datum). Error bars show $\pm 2 / \Delta x$ (blue circles: $25 \times 25$-cm cortex) and $\pm 1 / \Delta x$ (red crosses: $6 \times 6$-cm cortex), where $\Delta x=1 / L_{x}$ is the resolution limit of the spatial Fourier transform on an $L_{x}=L_{y}$ square grid. Crosses labeled (a)-(d) correspond to the four spectra of Fig. 13. 
fundamental unit of cortical organization [49]. Apparent cortical hexagonal symmetry has inspired others to theorize on the likely structure and logistics of memory development. Calvin suggests memories are stored on honeycomb-like structures, quasihexagonal tessellating activated regions [50] evolving via a Darwinian mechanism. The quasiperiodic orientation maps observed in primate cortex may be generated by Moiré interference between offset hexagonal mosaics of retinal cells, giving rise to hexagonally symmetric patterns on a larger spatial scale [51], but this suggestion remains contentious [52].

We must acknowledge that these examples from the literature are typically referring to the organization of minicolumns containing some hundreds of neurons, whereas our nucleation process yields much larger aggregates. However, we emphasize that spinodal decomposition provides an unforced organizing principle by which quasihexagonal patterning can emerge. In Sec. III F we showed how, with appropriate scaling of model parameters, centimeter-scale nucleation patterns (Fig. 6) could be replaced by millimeter-scale activity patterns (Fig. 13). Specifically, the simulations predict that smallerscale patterns can emerge in the model cortex, provided there are balanced reductions in both inhibitory diffusive strength $\left(D_{2}\right)$ and excitatory axonal range $\left(\Lambda_{e b}^{-1}\right)$. Thus subregions of the cortex that can be modelled as approximately homogeneous and isotropic - and that have modest gap-junction diffusion and axonal range-can support fine-scale spinodal patternings. However, these millimeter-scale patterns are probably too small to be resolved with the present generation of functional magnetic resonance imaging machines.

Our model is inherently static with only low-intensity white noise acting to perturb cortical activity, whereas the human cortex is exposed to a constantly changing spatiotemporal flux of incoming nonrandom and/or structured stimuli from both subcortical and long-distance cortical sources. This means that only the fastest-developing spinodal patterns are likely to be visible before being washed out by new patterns evoked by subsequent input stimuli. From the timedevelopment traces of Fig. 7, we might expect to see, in a real cortex, the bloblike primordial patterns of precipitation and early differentiation but not the fully ripened forms that evolve over tens of seconds. There is evidence that emergence of conscious awareness (e.g., recognition of a person or object) takes around $\sim 0.3$ to 0.6 s (e.g., Ref. [53]), and these timescales are entirely consistent with the $\sim 0.4$ - to 1.0 -s transition between "precipitation" and early "differentiation" for our spinodal patterns.

With the exception of the soliton simulation, the present spinodal model is simplified and static: We place the homogeneous cortex at an unstable separatrix and allow primordial activity patterns to precipitate, differentiate, and then slowly mature over tens of seconds under the influence of continuous low-intensity spatiotemporal white noise. Absent from the model is the notion that the conscious brain changes state relentlessly, so the patterns would not be expected to fully mature. Also, we have not attempted to design a homeostasis rule that would keep the cortex close to the spinodal zone within the binodal region and permit repeated cycling among the quench, decomposition, and annealing phases. In addition, in future work we would need to explore the effect of small fluctuations in $(E, I)$ drive and to quantify to what extent the nucleation grid can track changes in stimulus position.

The spontaneous dynamics that emerges in Fig. 10 when a weakly damped Hopf instability_induced by a slowed inhibitory synaptic response-interacts with spinodal decomposition flows is remarkable and unexpected. The selforganization into a soliton shockwave may represent a propagating population spike as seen in the brain-slice measurements shown in Fig. 11, believed to be a traveling-wave precursor characteristic of interictal activity and signaling proximity to a seizure state. Previous modeling has identified the Hopf instability as a source of the coherent slow waves observed during seizure [14,39]. Thus a slight adjustment of model parameters can take the system from soliton (interictal waves) to a global Hopf (full seizure).

In summary, spinodal decomposition has not previously been investigated as an agent for cortical pattern formation. Given appropriate initial conditions, and sufficient inhibitory gap-junction diffusivity, hexagonally symmetric activation patterns can emerge spontaneously from the homogeneous equilibrium state. Our preliminary findings indicate that a wide diversity of patterns is supported (e.g., Fig. 12) and that spinodal decomposition provides an alternative mechanism underpinning formation of cortical representations.

\section{APPENDIX: DETAILS FOR MOUSE BRAIN-SLICE MEASUREMENTS}

Following ethics approval from the University of Waikato Animal Ethics Committee, a 400- $\mu \mathrm{m}$ coronal neocortical slice was extracted from an adult male C57 wild-type mouse. The slice was prepared in carbogenated $\left(95 \% \mathrm{O}_{2}, 5 \% \mathrm{CO}_{2}\right)$ "normal" artificial cerebrospinal fluid (aCSF) and then perfused $(5 \mathrm{ml} / \mathrm{min})$ with carbogenated "zero-magnesium" aCSF during extracellular field potential recording of population activity; see Table II for aCSF chemical components and concentrations. The elimination of $\mathrm{Mg}^{2+}$ ions in the perfusate provides a proconvulsant function that compensates for the severed connections and consequent lack of excitatory stimulus entering the cortical slice [54]. Spontaneous field potential events were recorded with five $75-\mu \mathrm{m}$-diameter silver-silver chloride electrodes positioned in the cerebral cortex. Field potential signals were amplified $(\times 1000$ gain $)$, conditioned with 1-kHz low-pass and 1-Hz high-pass analog filters (A-M Systems, Carlsborg), and then sampled at $10 \mathrm{kHz}$ per channel (CED, Cambridge, England) for storage and analysis.

TABLE II. Chemical concentrations for artificial CSF solutions $(\mathrm{mM})$.

\begin{tabular}{lll}
\hline \hline Compound & Normal & Zero- $\mathrm{Mg}^{2+}$ \\
\hline $\mathrm{NaCl}$ & 125 & 124 \\
$\mathrm{KCl}$ & 2.5 & 5.0 \\
$\mathrm{MgCl}$ & 2.0 & - \\
$\mathrm{CaCl}_{2}$ & 1.0 & 2.0 \\
$\mathrm{NaH}_{2} \mathrm{PO}_{4}$ & 2.0 & 1.25 \\
$\mathrm{NaHCO}_{3}$ & 1.25 & 26 \\
$\mathrm{D}-$ glucose & 26 & 10 \\
\hline \hline
\end{tabular}


[1] A. M. Turing, The chemical basis of morphogenesis, Philos. Trans. R. Soc. London B 237, 37 (1952).

[2] P. Glansdorff and I. Prigogine, Thermodynamic Theory of Structure, Stability, and Fluctuations (Wiley-Interscience, John Wiley \& Sons, New York, 1974).

[3] L. Edelstein-Keshet, Mathematical Models in Biology (Random House, New York, 1988).

[4] L. Yang, M. Dolnik, A. M. Zhabotinsky, and I. R. Epstein, Pattern formation arising from interactions between Turing and wave instabilities, J. Chem. Phys. 117, 7259 (2002).

[5] H. R. Wilson and J. D. Cowan, A mathematical theory of the functional dynamics of cortical and thalamic nervous tissue, Kybernetik 13, 55 (1973).

[6] G. B. Ermentrout and J. D. Cowan, Large scale spatially organized activity in neural nets, SIAM J. Appl. Math. 38, 1 (1980).

[7] G. B. Ermentrout, Neural networks as spatio-temporal patternforming systems, Rep. Progr. Phys. 61, 353 (1998).

[8] P. C. Bressloff, New Mechanism for Neural Pattern Formation, Phys. Rev. Lett. 76, 4644 (1996).

[9] V. K. Jirsa and J. A. S. Kelso, Spatiotemporal pattern formation in neural systems with heterogeneous connection topologies, Phys. Rev. E 62, 8462 (2000).

[10] C. R. Laing and W. C. Troy, Two-bump solutions of Amaritype models of neuronal pattern formation, Physica D 178, 190 (2003).

[11] A. Hutt, M. Bestehorn, and T. Wennekers, Pattern formation in intracortical neuronal fields, Netw. Comput. Neural Syst. 14, 351 (2003).

[12] E. Negahbani, D. A. Steyn-Ross, M. L. Steyn-Ross, M. T. Wilson, and J. W. Sleigh, Noise-induced precursors of state transitions in the stochastic Wilson-Cowan model, J. Math. Neurosci. 5, 9 (2015).

[13] M. L. Steyn-Ross, D. A. Steyn-Ross, M. T. Wilson, and J. W. Sleigh, Gap junctions mediate large-scale Turing structures in a mean-field cortex driven by subcortical noise, Phys. Rev. E 76, 011916 (2007).

[14] M. L. Steyn-Ross, D. A. Steyn-Ross, and J. W. Sleigh, Interacting Turing-Hopf Instabilities Drive Symmetry-Breaking Transitions in a Mean-Field Model of the Cortex: A Mechanism for the Slow Oscillation, Phys. Rev. X 3, 021005 (2013).

[15] T. Fukuda, T. Kosaka, W. Singer, and R. A. W. Galuske, Gap junctions among dendrites of cortical GABAergic neurons establish a dense and widespread intercolumnar network, J. Neurosci. 26, 3434 (2006).

[16] C. Wozny and S. R. Williams, Specificity of synaptic connectivity between layer 1 inhibitory interneurons and layer $2 / 3$ pyramidal neurons in the rat neocortex, Cereb. Cortex 21, 1818 (2011).

[17] J. W. Cahn, On spinodal decomposition, Acta Metall. 9, 795 (1961).

[18] J. W. Cahn, Phase separation by spinodal decomposition in isotropic systems, J. Chem. Phys. 42, 93 (1965).

[19] F. S. Bates and P. Wiltzius, Dynamics of fluctuations and spinodal decomposition in polymer blends, J. Chem. Phys. 72, 4756 (1980).

[20] F. S. Bates and P. Wiltzius, Spinodal decomposition of a symmetric critical mixture of deuterated and protonated polymer, J. Chem. Phys. 91, 3258 (1989).
[21] R. A. L. Jones, L. J. Norton, E. J. Kramer, F. S. Bates, and P. Wiltzius, Surface-Directed Spinodal Decomposition, Phys. Rev. Lett. 66, 1326 (1991).

[22] F. Liu, M. Mondello, and N. Goldenfeld, Kinetics of the Superconducting Transition, Phys. Rev. Lett. 66, 3071 (1991).

[23] M. Plapp and J.-F. Gouyet, Dendritic growth in a mean-field lattice gas model, Phys. Rev. E 55, 45 (1997).

[24] M. V. Maximov, A. F. Tsatsul'nikov, B. V. Volovik, D. A. Bedarev, A. E. Zhukov, A. R. Kovsh, N. A. Maleev, V. M. Ustinov, P. S. Kop'ev, Zh. I. Alferov, R. Heitz, N. N. Ledentsov, and D. Bimberg, Quantum dots formed by activated spinodal decomposition of $\mathrm{InGa}(\mathrm{Al}) \mathrm{As}$ alloy on InAs stressors, Physica E 7, 326 (2000)

[25] J. A. Witt, D. R. Mumm, and A. Mohraz, Bijel reinforcement by droplet bridging: A route to bicontinuous materials with large domains, Soft Matter 9, 6773 (2013).

[26] M. L. Steyn-Ross, D. A. Steyn-Ross, J. W. Sleigh, and D. T. J. Liley, Theoretical electroencephalogram stationary spectrum for a white-noise-driven cortex: Evidence for a general anesthetic-induced phase transition, Phys. Rev. E 60, 7299 (1999).

[27] M. V. Bennett and R. S. Zukin, Electrical coupling and neuronal synchronization in the mammalian brain, Neuron 41, 495 (2004).

[28] D. A. Steyn-Ross, M. L. Steyn-Ross, and J. W. Sleigh, Equilibrium and nonequilibrium phase transitions in a continuum model of an anesthetized cortex, in Neural Fields: Theory and Applications, edited by P. beim Graben, S. Coombes, R. Potthast, and J. J. Wright (Springer-Verlag, Berlin, 2014), Chap. 15 , pp. $393-416$

[29] P. A. Robinson, C. J. Rennie, and J. J. Wright, Propagation and stability of waves of electrical activity in the cerebral cortex, Phys. Rev. E 56, 826 (1997).

[30] L. E. Reichl, A Modern Course in Statistical Physics, 2nd ed. (John Wiley \& Sons, New York, 1998).

[31] J. W. Cahn and J. E. Hilliard, Free energy of a nonuniform system. I Interfacial free energy, J. Chem. Phys. 28, 258 (1958).

[32] M. Hillert, A solid-solution model for inhomogeneous systems, Acta Metall. 9, 525 (1961).

[33] H. E. Cook, Brownian motion in spinodal decomposition, Acta Metall. 18, 297 (1970).

[34] V. P. Skripov and A. V. Skripov, Spinodal decomposition (phase transitions via unstable states), Sov. Phys. Usp. 22, 389 (1979).

[35] See Supplemental Material at http://link.aps.org/supplemental/ 10.1103/PhysRevE.99.012318 for simulation movies showing formation of honeycomb, meander, nucleation, and soliton activation patterns.

[36] M. Fiałkowski and R. Hołyst, Dynamics of phase separation in polymer blends revisited: Morphology, spinodal, noise, and nucleation, Macromolec. Theory Simul. 17, 263 (2008).

[37] M. L. Steyn-Ross, D. A. Steyn-Ross, M. T. Wilson, and J. W. Sleigh, Modeling brain activation patterns for the default and cognitive states, NeuroImage 45, 298 (2009).

[38] M. L. Steyn-Ross, D. A. Steyn-Ross, J. W. Sleigh, and M. T. Wilson, A mechanism for ultra-slow oscillations in the cortical default network, Bull. Math. Biol. 73, 398 (2011).

[39] M. L. Steyn-Ross, D. A. Steyn-Ross, and J. W. Sleigh, Gap junctions modulate seizures in a mean-field model of general anesthesia for the cortex, Cogn. Neurodyn. 6, 215 (2012). 
[40] C. E. Stafstrom, Distinct mechanisms mediate interictal and pre-ictal discharges in human temporal lobe epilepsy, Epilepsy Curr. 11, 200 (2011).

[41] G. Huberfeld, L. Menendez de la Prida, J. Pallud, I. Cohen, M. Le Van Quyen, C. Adam, S. Clemenceau, M. Baulac, and R. Miles, Glutamatergic pre-ictal discharges emerge at the transition to seizure in human epilepsy, Nat. Neurosci. 14, 627 (2011).

[42] P. H. C. Eilers, A perfect smoother, Anal. Chem. 75, 3631 (2003).

[43] S. Amari, Dynamics of pattern formation in lateral-inhibition type neural fields, Biol. Cybern. 27, 77 (1977).

[44] R. Ben-Yishai, R. L. Bar-Or, and H. Sompolinsky, Theory of orientation tuning in visual cortex, Proc. Natl. Acad. Sci. USA 92, 3844 (1995).

[45] Y. Burak and I. R. Fiete, Accurate path integration in continuous attractor network models of grid cells, PLoS Comput. Biol. 5, e1000291 (2009).

[46] V. B. Mountcastle, Perceptual Neuroscience: The Cerebral Cortex (Harvard University Press, Cambridge, MA, 1998).

[47] O. V. Favorov and M. E. Diamond, Demonstration of discrete place-defined columns-segregates-in the cat SI, J. Comp. Neurol. 298, 97 (1990).
[48] O. V. Favorov and D. G. Kelly, Minicolumnar organization within somatosensory cortical segregates: I. Development of afferent connections, Cereb. Cortex 4, 408 (1994).

[49] V. B. Mountcastle, Modality and topographic properties of single neurons of cat's somatic sensory cortex, J. Neurophysiol. 20, 408 (1957).

[50] W. H. Calvin, Cortical columns, modules, and Hebbian cell assemblies, in The Handbook of Brain Theory and Neural Networks, edited by M. A. Arbib (Bradford Books/MIT Press, Cambridge, MA, 1995), pp. 269-272.

[51] Se-Bum Paik and D. L. Ringach, Retinal origin of orientation maps in visual cortex, Nat. Neurosci. 14, 919 (2011).

[52] V. R. A. Hore, J. B. Troy, and S. J. Eglen, Parasol cell mosaics are unlikely to drive the formation of structured orientation maps in primary visual cortex, Vis Neurosci. 29, 283 (2012).

[53] R. Q. Quiroga, L. Reddy, G. Kreiman, C. Koch, and I. Fried, Invariant visual representation by single neurons in the human brain, Nature 435, 1102 (2005).

[54] A. Stepanyants, L. M. Martinez, A. S. Ferecskó, and Z. F. Kisvárday, The fractions of short- and long-range connections in the visual cortex, Proc. Natl. Acad. Sci. USA 106, 3555 (2009). 